\title{
HUNGER, W
}

Ueber die funktion der oberflaechlichen schleimbildungen im pflanzenreiche. 1899. 


\section{UEBER DIE FUNKTION DER OBERFLECHLICHEN SCHLEIMBILDUNGEN IM PFLANZENREICHE}

\section{INAUGURAL-DISSERTATION}

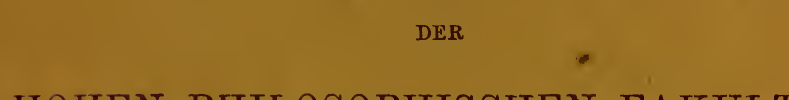

HOHEN PHILOSOPHISCHEN FAKULT ATT

DER

GESAMT-UNIVERSITET JENA

ZUR

\section{ERLANGUNG DER DOKTORWUERDE}

VORGELEGT VON

WILHELM HUNGER
GEBOREN ZU AMSTERDAM, HOLLAND

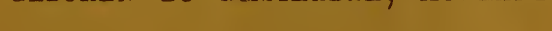

$\rightarrow+\infty$

BUCHHANDLUNG UND DRUCKEREI

E. J. BRILL LEIDEN, 1899 

UEBER DIE FUNKTION IOER OBERFLACHLICHEN SCHLEIMBILDUNGEN IM PFLANZENREICHE 
Genehmigt von der philosophischen Fakultät der Universität Jena auf Antrag des Herrn Professor Dr. Stahi.

Jena, den 2. August 1898.

Geh. Hofrath Prof. Dr. Thomae,

d. Z. Dekan. 
UEBER DIE FUNKTION DER OBERFLECHLICHEN SCHLEIMBILDUNGEN IM PFLANZENREICHE

\section{INAUGURAL-DISSERTATION}

DER

HOHEN PHILOSOPHISCHEN FAKULTAT

DER

GESAMT-UNIVERSITET JENA

ZUR

ERLANGUNG DER DOKTORWUERDE

VORGELEGT VON

\section{WILHELM HUNGER}

GEBOREN ZU AMSTERDAM, HOLLAND

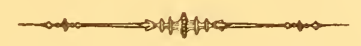

BUCHHANDLUNG UND DRUCKEREI

F. J. BRIL工

LEIDEN, 1899 


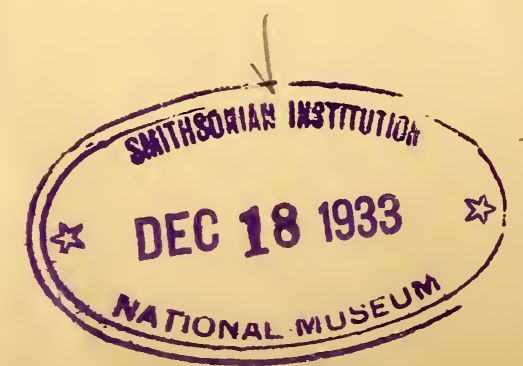


Dem Andenken

\section{MEINER TEUERN ELTERN}

Gewidmet. 

INHALT.

\begin{tabular}{|c|c|c|c|c|c|c|}
\hline & & & & & & Seite \\
\hline Einle & & . . . . . . . . . . . & & & & - 1. \\
\hline Specie & Teil & . . . . . . . . . . & . & & & 7. \\
\hline I. & chnitt. & Schleimbildung bei freibeweglichen Pfla & $\operatorname{lanz}$ & & & \\
\hline II. & » & 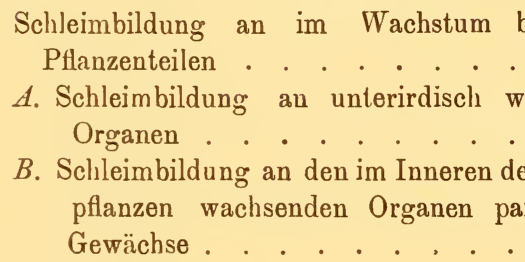 & 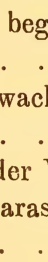 & isen & & \\
\hline III. & " & $\begin{array}{l}\text { Schleimbildung bei den Bryophyten } \\
\text { Schleimbildung bei den Hepaticae }\end{array}$ & & & & \\
\hline IV. & " & $\begin{array}{l}\text { Schleimbildung bei den Pteridophyten. } \\
\text { Schleimbildung bei den Filices . }\end{array}$ & & & & \\
\hline V. & " & $\begin{array}{l}\text { Schleimbildung bei den Laubknospen } \\
\text { A. Laubknospen der Dicotyledonen } \\
\text { B. Laubknospen der Monocotyledonen }\end{array}$ & & & & \\
\hline VI. & " & Schleimbildung bei den Wasserpflanzen & & $\cdot$ & & 60 . \\
\hline VII. & " & Schlussbemerkungen . & & • & & 70. \\
\hline Litte & Ver & ichniss . . . . . & & & & \\
\hline
\end{tabular}




\section{E I N L E I T U N G.}

Die Frage nach der Funktion der oberfächlichen Schleim- und Gallertbildungen, welche in so mannigfacher Weise im Pflanzenreiche auftreten, ist sehr verschiedenartig beantwortet worden.

Es dürfte daher durchaus gerechtfertigt sein, die verschieden lautenden Auffassungen gegen einander abzuwägen und an der Hand neuer Versuche auf ihren Werth zu prüfen.

Von den zahlreichen Arbeiten, die von den hier allein berücksichtigten oberflächlichen Schleimbildungen handeln, verdient in erster Linie die im Jahre 1868 erschienene Abhandlung von HaNsTEIN ${ }^{1}$ ) genannt $\mathrm{zu}$ werden.

1) Ueber die Organe der Harz- und Schleimabsonderung in den Laubknospen. Bot. Zeit., 1868, $n^{\circ}$. 43-46. Taf. XI-XII. 
Hanstein untersuchte eingehend eine grosse Anzahl von Laubknospen und fand, dass bei vielen eine reichliche Bekleidung mit schleimbildenden Trichomen vorkommt, welchen er den Namen von Schleimzotten oder Colleteren ${ }^{1}$ ) gab, da sie nicht allein in ihrer Verrichtung, sondern auch in ihrer Gestalt einigermaassen an die sogenannten Zotten im tierischen Darmkanal erinnern sollen. Hansteis beschränkt sich nicht auf morphologische und anatomische Angaben über diese Schleimzotten, sondern spricht auch seine Meinung über die Funktion derselben aus. Er kommt hierbei zu dem Schlusse, dass die reichliche Bekleidung mit Schleimzotten auf vielen Knospen einen Schutzapparat darstellt zur Verminderung der Ausdünstung, zur Erhöhung der Turgescenz und somit zur Begünstigung der Entwickelung des Knospenteiles $\left.{ }^{1}\right)$.

Im Jahre 1886 ist es darauf GoebeLen, welcher in einer Arbeit: „Ueber die Schutzvorrichtungen am Stammscheitel der Farne“ ${ }^{2}$ ) auch die dort von ihm angetroffene Schleimbildung bespricht, deren Bedeutung er in der Fähigkeit sucht, die Transpi-

1) L. c., pag. 700 .

2) Flora, 1886, $\mathrm{n}^{\circ}, 29-31$. Taf. XI. 
ration zu vermindern und Wasser zu dem Zwecke aufzusaugen, um es entweder zu speichern oder sofort den jungen. Geweben zuzuführen ${ }^{1}$ ).

Im Jahre 1858 spricht dann STAHL in seiner Arbeit: „Pflanzen und Schnecken“ 2) seine Auffassung dahin aus, dass Schleim- und Gallertüberzüge wirksame Schutzmittel gegen die Angriffe von pflanzenfressenden Tieren, speciell Schnecken, darstellen.

Zuletzt haben wir noch die Gozbes'sche Auffassung, welche von ihm in verschiedenen Schriften und auch von mehreren seiner Schüler (KÜHN, RAcıBoRsKI, Rtge, Schlluing) vertreten worden ist. Während Hanstein und Goebeler nur Landpflanzen berücksichtigen, und andererseits STAHL's Angaben sowohl für Land- als für Wasserpflanzen gelten, schreibt Goeber ${ }^{3}$ ) dem Schleim eine verschiedene Bedeutung zu, je nachdem er mit der Luft oder mit Wasser in Berührung tritt.

Im ersteren Fall sucht Goebel seine Bedeutung darin, dass er Schutz gegen das Vertrocknen gewähre; bei Wasserpflanzen soll dagegen die Bedeutung der Schleimhülle darin liegen, „dass

1) L. c., pag. $483-486$.

2) Jena, 1888.

3) Pflanzenbiologische Schilderungen, Marburg, 1889-1893, I. II. 
dieselbe Wasser sehr festhält und so den Hindurchtritt von Wasser erschwert".

Hiermit habe ich die wichtigsten Ansichten über die biologische Bedeutung des Schleimes kurz hervorgehoben; ich werde an verschiedenen Stellen Gelegenheit haben, auf die speciellen Auffassungen noch näher einzugehen.

Bevor ich aber an den „Speciellen Teil“ herantrete, möchte ich zuletzt noch einen allgerneinen biologischen Gesichtspunkt hervorheben, der sich mir während dieser Arbeit immer wieder aufdrängte und in treffender Weise durch RACIвоRSкI ausgedrückt worden ist. Ich gebe hier wörtlich wieder, was RAciborski im Jahre 1895 in seiner Arbeit: „Ueber die Schutzvorrichtungen der Blüthenknospen " ${ }^{1}$ ) in dieser Hinsicht sagt: "Nicht nur an den ausgebildeten Blüthen sollen wir die Anpassungen an ihre Funktionen bewundern, an jeder Entwickelungsstufe treten uns Anpassungen und Schutzvorrichtungen entgegen. Von den ersten Primordien angefangen, haben wir mit wechselnden Anpassungen zu thun und die Phasen der Entwicklung kann man biologisch als Ergebnisse

1) Flora, 1895, Ergänzungsbd., pag. 162. 
eines Compromisses zwischen den augenblicklich thätigen Anpassungen und den erst später im Verlaufe der Entwickelung zur Geltung kommenden betrachten. Auch die Entwickelungsgeschichte fordert eine biologische Betrachtung".

Eine Pflanze adaptirt sich also mit ihren biologischen Eigenschaften nicht bloss den äusseren Bedingungen, sondern die biologischen Eigenschaften selber passen sich ihrerseits dem immer im Wechsel begriffenen Lebensprocess an, sodass dadurch ein Funktionswechsel einer biologischen Eigenschaft herbeigeführt werden kann.

Damit in Verbindung kann es geschehen, dass solche Eigenschaften, welche zuerst eine Nebenfunktion im Leben einer Pflanze einnehmen, in ihrer biologischen Bedeutung fortschreiten in Beziehung zu folgenden Entwickelungsphasen, und zwar so weit selbst, dass sie in ihren Leistungen einer ehemaligen Hauptfunktion gleichwertig werden können.

Auf der Basis dieses Gedankens bauen sich die folgenden Untersuchungen über die biologische Bedeutung der oberflächlichen Schleimbildung auf.

$\mathrm{Zu}$ diesen Untersuchungen wurde nur frisches, 
zum Teil von mir selbst gesammeltes, zum grössten Teil jedoch schon seit mehreren Jahren im hiesigen botanischen Garten kultiviertes Material verwandt. Die Arbeit wurde im botanischen Institut hierselbst angefangen und vollendet, und spreche ich am Schlusse dieser Einleitung meinem hochverehrten Lehrer, Herrn Professor Dr. STAHL, für das meiner Arbeit gewidmete Interesse sowie für vielfache Unterstützung und Anregung meinen herzlichsten Dank aus. 


\section{SPECIELLER TEIL.}

Die im Pflanzenreich sehr verbreiteten oberflächlichen Schleimbildungen können wir zum Zweck unserer Untersuchungen unter folgende drei Kategorien bringen:

Die im Wasser lebenden oder doch an ein nasses Substrat gebundenen freibeweglichen Pflanzen lassen oberflächliche Schleim- resp. Gallertbildungen erkennen, welche hier in direkter Berührung mit dem Wasser stehen.

Ein zweiter Fall ist der, wo bei im Wachstum begriffenen Pflanzen oberflächliche Schleimbildung an verschiedenen Teilen auftritt. Dieselbe kann nun aber hier in Berührung treten entweder mit einem nicht zur Pflanze gehörenden fremden Gegenstand 
(Boden, Wirthspflanze), oder mit Teilen resp. Organen derselben Pflanze.

Im dritten Falle kann die oberflächliche Schleimbildung sowohl mit Wasser, als auch mit anderen, der Pflanze selbst zugehörenden Teilen in Berührung treten. 


\section{ABSCHNITT.}

\section{Schleimbildung bei freibeweglichen Pflanzen.}

Die Durchsicht der Litteratur und eigene Untersuchungen haben mir gezeigt, dass überall da, wo Freibeweglichkeit bei pflanzlichen Organismen mit zarter Oberfläche vorkommt, die Anwesenheit von Schleim resp. Gallerte konstatiert werden kann. Dieser Umstand weist darauf hin, dass intime Beziehungen zwischen freier Bewegung und Schleimresp. Gallertbildung vorhanden sind.

Das Vorkommen, von schleimigen oder gallertigen Ueberzügen ist bei den Algen sehr verbreitet. Den höchst interessanten Untersuchungen von KLEBS ${ }^{1}$ ),

1) Ueber die Organisation der Gallerte bei einigen Algen und Flagellaten, in Unters. aus d. botan. Instit. zu Tübingen, Bd. III, pag. 333-418. T'af. IJI-IV. 
HaUPTFleisch $^{1}$ ) u. A. verdanken wir unsere jetzigen Kenntnisse über Beschaffenheit und Eigenschaften dieser Bildungen. KLebs hebt an einer Stelle ${ }^{2}$ ) die Möglichkeit hervor, dass die Gallerthülle im Dienste der Ernährungsfunktion stehen könnte; doch weist er selbst diesen Gedanken als nicht berechtigt zurück. An einer anderen Stelle ${ }^{3}$ ) sagt er in Betreff gewisser Desmidiaceen wörtlich: „Eine sehr wichtige und merkwürdige Eigenheit besteht in der Schleimbildung während der Bewegung. Das Emporsteigen über das Substrat geschieht nur dadurch, dass das unter dem Substrat anfangs aufsitzende Ende der Zelle allmählich einen Schleimfaden ausscheidet, auf welchem sich die Alge erhebt. Ohne Schleimfaden ist nach meinen bisherigen Beobachtungen ein Emporsteigen im Wasser nicht möglich; ein freies Schwimmen habe ich noch von keiner Desmidiacee nachweisen können".

ScHRÖTER ${ }^{4}$ ) schreibt der Gallerte die Bedeutung eines

1) Zellmembran und Hüllgallerte der Desmidiaceae, Greifswald, 1888. (Inaug.-Dissert.)

2) L. c., pag. 360.

3) Ueber Bewegung und Schleimbildung der Desmidiaceae, im Biol. Centralbl., 1885, Bd. V, n ${ }^{\circ}$ 12, pag. 355 .

4) Die Schwebeflora unserer Seen (Das Phytoplankton), im 99. Neujahrsblatt d. Naturforsch. Gesellsch. in Zürich, 1897, pag. 51. 
Schwebemittels $\mathrm{zu}$, worin wir ihm jedoch nicht beipflichten können, da ja, wie Schüтт ${ }^{1}$ ) gezeigt hat, das Schweben durch ganz andere Einrichtungen erleichtert wird. Will man zu einer richtigen Auffassung über die Bedeutung der Gallerte bei den Algen gelangen, so ist dies meiner Ansicht nach nur dadurch möglich, dass man auch die Schleimbildungen anderer, im Wasser lebender Organismen zum Vergleich heranzieht. Ich werde mich deshalb nicht bloss auf die pflanzlichen Organismen beschränken, sondern auch die im Wasser lebenden Tiere in den Kreis meiner Betrachtung ziehen, um so hoffentlich auf dem Wege des Vergleiches zu einheitlichen und den Thatsachen entsprechenden Schlüssen $\mathrm{zu}$ gelangen.

Stellt man sich die Frage nach der Bedeutung der Schleimabsonderung an der Oberfläche des Fischkörpers, so wird man ihr wohl in erster Linie die Rolle eines Schutzmittels gegen Verletzungen zuschreiben. Hätte das Tier keinen Schleimüberzug, dann würde es bei den beim Schwimmen unvermeidlichen Berührungen mit seinen Weg kreuzenden

1) In Engler und Prantu, Natürl. Pflanzenfamilien, 1896, Lief. 143-145, pag. 37 . 
rauhen Gegenständen leicht Verletzungen erleiden. Da nun aber die Oberfläche des Körpers durch den Schleim glatt und schlüpfrig gemacht ist, so wird er beim Anstossen abgleiten, ohne von der Berührung Nachteil zu erleiden. Das Leben eines Aales zwischen Steinen und anderen harten Gegenständen seiner Aufenthaltsorte wäre sicher unmöglich, wenn seine Haut nicht eine so mächtige Schleimschicht bedeckte. Dass diese Letztere auch, in Folge der geringeren Reibung der Körperoberfläche mit dem Wasser, für die Fortbewegung vorteilhaft sein muss, ist wohl kaum zu bestreiten.

Auch Goeber ${ }^{1}$ ) zieht an einer Stelle, zur Stütze seiner oben (S. 3-4) erwähnten Ansicht, wonach Schleimüberzüge das Eindringen von im Wasser gelösten Salzen erschweren sollen, den Aal herbei, indem er sich auf eine Notiz bei Regnadd ${ }^{2}$ ) beruft, wonach die Ueberführung von Aalen aus dem Süsswasser in Salzwasser schädlich, ja selbst tötend wirkt, wenn man vorher die Schleimhülle vom Körper abgerieben hat.

Gehen wir jetzt zu den Algen über und be-

1) Pflanzenbiolog. Schilderungen, 1891, II, pag. 237.

2) La vie dans les eaux, pag. 438. 
trachten wir zunächst diejenigen, welche die Fähigkeit besitzen, sich activ zu bewegen. Hier kommen unter anderen in Betracht die Cyanoplyceen (Oscillarien), Diatomeen, Desmidiaceen und Conjugaten (Spirogyren), bei denen allen eine Gallerthülle in grösserer oder geringerer Mächtigkeit vorhanden ist.

Die kriechende Bewegung der Oscillarien über ihr Substrat ist meist keine gleichmässige, sondern eine ruckweise vor sich gehende.

Die active Bewegung, welche bei Oscillarien auch den ausgewachsenen Fäden zukommt, findet sich bei anderen Cyanophyceen nur bei den beweglichen Hormogonien. Mit der Bewegungsfähigkeit ist anch hier stets das Vorhandensein einer Gallerthülle verbunden; dagegen kommt es vor, dass, wenn die Bewegung der Hormogonien, welche nur von kurzer Dauer ist, verloren gegangen und dieselben zum neuen Thallus auswachsen, die Gallerthülle zugleich abgestossen wird.

Die Diatomeen sind entweder freibeweglich oder festgewachsen; die freibeweglichen wieder schweben teils frei im Wasser (Planktondiatomeen), teils haften sie an dem Substrat (Boden, Steinen, Wasserpflanzen) (Grunddiatomeen). Die freien Grunddiatomeen besitzen active Bewegung, und hier ist die Gal- 
lerthülle auch immer deutlich nachweisbar. Durch die Untersuchungen von KLEBAнN ${ }^{1}$ ) wissen wir, dass die Gallertkappen auf den Kielen sitzen, und zwar gerade da, wo die Letzteren an den Zellenenden sich hakenförmig umbiegen. Jede Zelle hat also zunächst vier Kappen, je eine an jedem Ende jeder Panzerhälfte, also an der Stelle, wo bei der Bewegung in erster Linie das Anstossen an fremde Gegenstände erfolgen kann.

Die Bedeutung, welche wir der Gallerthülle der Oscillarien und der Hormogonien anderer Cyanophyceen, wie auch den Gallertkappen der Diatomeen zuschreiben möchten, wäre also identisch mit derjenigen der Schleimüberzüge der Fische. Voran steht der Schutz gegen mechanische Verletzungen. So vermag der Oscillarien-Faden an den dem Substrat aufiuhenden Gegenständen vorüberzugleiten, ohne durch die Berührung mit denselben verletzt zu werden; die Gallertkappen an den Kielen der Panzerhälften der Diatomeen wirken gegen oft genug eintretende Quetschungen wie Puffer.

Welche Rolle Gallertüberzüge als Schutzmittel

1) Beiträge zur Kenntniss der Auxosporenbildung, in Pringshein's Jahrbüch., 1896, Bd. 29, pag. 595-652. Taf. X. 
gegen Tierfrass übernehmen, ergiebt sich aus den Beobachtungen von SтAнL ${ }^{1}$ ), welcher direkt wahrnehmen konnte, wie Wasserschnecken vergebliche Versuche machten, die langen Internodialzellen von Nitella syncarpa anzufressen. Die Radula glitt an der festen Gallerte ab, ohne dass ihre Zähne einzureissen vermochten.

Dasselbe konnte ich konstatieren bei Chaetophora pissiformis. Ich fand diese Alge in der Umgebung von Jena, in einem Tümpel, in welchen der gemeine Flohkrebs (Gammarus pulex L.) massenhaft anwesend war.

An diesem Standorte war keine andere Wasserpflanze als die Chaetophora zu finden, sodass angenommen werden darf, dass keine andere Alge im Stande war, sich den gefrässigen Tieren gegenüber zu halten.

Die nıächtige Gallerthülle der Chaetophora-Büschel macht eben dem Gammarus das Anbeissen unmöglich.

Auch zartere, mit Gallertscheiden versehene Algen werden durch diese gegen die Angriffe kleinerer Tiere geschützt. Versucht man, selbst mit einer

1) L. c., pag. 83. 
scharfen Scheere, einen Spirogyra zu durchschneiden, so wird dies nur gelingen, wenn der Schnittversuch sehr rasch durchgeführt wird. Bei langsamerer Annäherung der beiden Scheerenhälften weicht der Faden zurück, ohne Schaden zu erleiden. Kleinere Tiere werden vergeblich versuchen, solche Fäden anzubeissen.

Durch die Arbeiten von $\mathrm{K}_{\mathrm{LEBS}}{ }^{1}$ ) sind wir über die Bewegungen der Desmidiaceen gut orientiert und wissen heute, dass die Fortbewegung nur möglich gemacht wird durch den Schleimstiel, welcher während der Bewegung ausgeschieden wird. Der Gallertbildung an der Oberfläche des Thallus der Desmidiaceen möchte ich aber, ebenso wie der Gallerthülle der Spirogyren, dieselbe Funktion zuschreiben, wie in den obengenannten Beispielen (Oscillarien und Diatomeen).

Wie steht es nun aber mit denjenigen Algen, welche keine active Bewegung zeigen und doch eine Gallerthülle besitzen?

Die Hauptbedeutung des Schleimes resp. der Gallerte ist nach unserer Auffassung bei allen Algen, mögen sie sich aktiv bewegen, oder passiv be-

1) L. c. 
wegt werden, oder ganz ohne Bewegung sein, zunächst zu suchen in dem Schutz gegen mechanische Verletzungen.

Bei Meeresalgen, wo die passive Bewegung besonders stark ist, findet sich die Schleimbildung in ganz allgemeiner Verbreitung. Wie aus den Untersuchungen von Goignard ${ }^{1}$ ) hervorgeht, vollzieht sich bei den Laminarien dieser Vorgang im Inneren der Pflanze.

Die gebildeten Schleimmassen gelangen dann zur Einhüllung des Thallus an die Oberfläche desselben. Bei den marinen Rhodophyceen erreichen die Schleimbildungen auch mächtige Dimensionen.

Bei der im Süsswasser vorkommenden Gattung Batrachospermum ist, wie bekannt, ebenfalls eine dicke Schleimhülle vorhanden.

In rasch fliessenden Bächen mit steinigem Grund wird diese Alge fortwährend hin und her geschleudert, und nur die Gallerthülle ermöglicht es ihr, die Folgen dieser Bewegungen ohne Schaden zu ertragen; denn ohne diesen Ueberzug würden die zartgebauten Fäden sofort verletzt werden.

1) Observations sur l'appareil mucifere des Laminariacées. Annales des Sciences natur., Paris, 1892, 'Tom. XV, pag. 1-46. 
W $_{\text {ILle }}{ }^{1}$ ) und LAgerheim ${ }^{2}$ ) haben schon früher darauf hingewiesen, dass durch die grosse Schlüpfrigkeit, welche viele Algen den Gallertüberzügen verdanken, die Reibung gegen das Wasser sehr reduciert wird, welcher Umstand dazu beiträgt, die Alge gegen Wegreissen zu schützen.

Der oft ausgesprochenen Ansicht, dass dieselben Bildungen als Schutzmittel gegen Austrocknen bei an die Luft gekommenen Algen dienen sollen, kann ich mich ebenso wenig wie Laglrheim (l. c.) anschliessen. Dieser Forscher sah die mit einer mächtigen Gallerthülle umgebenen Hydrurus-Exemplare rasch trocken werden und absterben, wenn sich das Wasser von ihnen zurückgezogen hatte.

Auch bei Spirogyren verlangsamt die Gallerthülle kaum das Absterben in Folge von Wasserverlust. Es ist einem Jeden bekannt, wie rasch trocken liegende Spirogyren-Fäden den Objektträgern fest ankleben; ja selbst in relativ feuchter Luft schrumpfen isolierte Fäden in kurzer Zeit, oft in weniger als einer Minute, vollständig ein.

1) Bidrag til Algernes physiologiske anatomi, pag. 39; vgl. Bot. Centralblatt, 1886.

2) Zur Entwickelungsgeschichte des Hydrurus. Berichte der Deutschen Bot. Gesellsch., 1888. 
Auf die Rolle, welche Gallert- und Schleimbildungen bei Algen noch nach anderen Seiten, z. B. als Haftstoff bei kolonienbildenden Chroococcaceen, Tetraspora u. s. w., spielen können, gehen wir hier nicht weiter ein und wenden uns zu den Plasmodien der Myxomyceten, speciell der Physareen, deren Schleimspuren jedermann bekannt sind.

Wie das Kriechen einer Schnecke über das Substrat begünstigt wird durch die Schleimschicht, welche vom Fuss ausgeschieden wird, so dient auch der Schleim, welcher beim Fortkriechen eines $M y$ xomyceten dessen Weg bezeichnet, als eine Art Schmiere, welche es dem zarten Plasmodium ermöglicht, ohne Schaden zu erleiden, über rauhe Gegenstände hinwegzukriechen. 


\section{ABSCHNITT.}

\section{Schleimbildung an im Wachstum begriffenen Pflanzenteilen.}

A. SCHLEIMBILDUNG AN UNTERIRDISCH WACHSENDEN ORGANEN.

Die Anwesenheit von Schleim an der Wurzelspitze und an den Wurzelhaaren ist schon lange bekannt. Im ersteren Fall entsteht der Schleim durch Desorganisation der äusseren Zellen der Wurzelhaube; bei den Wurzelhaaren ist es die äusserste Membranschicht, welche verschleimt.

Was für eine Funktion soll der Schleim nun hier zu erfüllen haben?

Wir stehen hier vor mehreren und sehr verschiedenartigen Ansichten. Darwin nimmt an, dass der Schleim den Zweck habe, die Wurzel mit den umgebenden Erdpartikelchen zu verkleben, um so das weitere Eindringen in den Boden zu unterstützen. 
Schwarz ${ }^{1}$ ) meint, dass die von der Wurzel ausgeschiedene Säure sich leicht in dieser Schleimschicht verbreiten und so lösend auf die Bodenpartikelchen wirken kann. Ausserdem soll der Schleim, nach seiner Meinung, den Wurzelhaaren einen gewissen Schutz vor zu schnellem oder plötzlichem Austrocknen gewähren. SACHS ${ }^{2}$ ) nimmt an, dass die glatte, schlüpfrige Oberfläche der festen Wurzelkappe bei ihrer konischen Form das Vordringen in den Boden erleichtert. Endlich schreibt GoebeL ${ }^{3}$ ) hierüber wörtlich: $„ . .$. die im Boden befindlichen und dort mit Wasser in Berührung tretenden Wurzelhaare der Landpflanzen besitzen gleichfalls eine solche Schleimhülle. Die Bedeutung dieser Scheimhülle liegt darin, dass dieselbe Wasser zähe festhält und so den Hindurchtritt des Wassers erschwert" u.s.w.

Diese letzte Ansicht ist, wie wir wissen, durchaus im Widerstreit mit den bisherigen Kenntnissen über Transpiration und Wasseraufnahme. Das Hauptresultat der Transpiration ist die Wasserbe-

1) Die Wurzelhaare der Pflanzen, in Unters. aus d. botan. Instit. zu Tübingen, 1883, Bd. I, Heft 2.

2) Vorlesungen über Pflanzen-Physiologie, 1882, pag. 20.

3) Pflanzenbiolog. Schilderungen, II, pag. 235-236. 
wegung in der Pflanze und damit die Zufuhr von Nährsalzen, welche durch die Wurzeln aufgenommen werden; ja, wir wissen selbst, dass specielle Organe, die Wasserspalten, dazu ausgebildet sind, diese Wasserbewegung auch während der Nacht und in den ersten Morgenstunden aufrecht zu erhalten, sodass es nicht wohl anzunehmen ist, dass Einrichtungen vorhanden sein sollten, welche auf der einen Seite verhindern, was auf der anderen Seite erzielt wird.

Dass der Schleim nicht die Fähigkeit besitzt, den Hindurchtritt des Wassers zu erschweren, darauf werde ich in einem späteren Abschnitt ausführlicher eingehen und citiere für jetzt nur eine Stelle aus der angeführten Abhandlung von Schwarz ${ }^{1}$ ), in der sich dieser folgendermaassen äussert: „Man könnte glauben, dass durch eine derartige Umhüllung des Wurzelhaares der Durchgang gelöster Stoffe erschwert würde; dies findet jedoch nicht statt. Wir erkennen dies aus der Leichtigkeit, mit der Farbstoffe, die zur inneren Celluloseschicht gelangten, beim Einlegen in Wasser wieder ausgewaschen wurden".

1) L. c., pag. 144 . 
Ein weiterer Beweis dagegen liegt noch in dem Umstande, dass an den Wurzeln von Wasserpflanzen die Schleimbildung meistens ganz unterbleibt und dass sogar in einigen wenigen Fällen die Wurzelhaube selbst ganz verloren geht, wie AmвronN und Westermaier ${ }^{1}$ ) es für Azolla caroliniana Link nachgewiesen haben ${ }^{2}$ ).

Es wird darum wohl eine andere Funktion in Betracht kommen, und zwar die, auf welche schon SAchs hingewiesen hat: das Vorhandensein der Schleimhülle an der Spitze der Wurzeln und an den Wurzelhaaren wirkt in ähnlicher Weise, wie bei manchen Tieren, zum Beispiel den Regenwürmern, nämlich als eine Art Schmiere, welche das Eindringen und Fortwachsen derselben erleichtert, inclem sie ihrer schlüpfrigen Oberfläche die Fähigkeit zu gleiten verleiht und ihnen einen nicht unbeträchtlichen Schutz gegen Verletzungen gewährt, denen die unterirdisch wachsenden Teile selbstverständlich oft genug ausgesetzt sind.

1) Ueber eine biologische Eigenthümlichkeit der Azolla caroliniana Link, in Abhandl. d. Botan. Vereins d. Provinz Brandenburg, Berlin, 1880, Jahrg. XXII, pag. 58-61. Taf. III.

2) Die Vermutung der beiden Forscher, dass diese biologische Erscheinung auch bei anderen Arten der Gattung Azolla vorkommen dürfte, fand ich im vorigen Sommer bei A. filicuoides Lam, bestätigt. 
Durch die Untersuchungen von van Tieghem und Douliot ${ }^{1}$ ) wissen wir, dass die Wurzelhaube entwickelungsgeschichtlich aus zwei gänzlich verschiedenen Teilen besteht. Den äusseren Haubenteil der Seitenwurzel nennt van Tieghem „la poche“, oder „la poche digestive“, weil er annimmt, dass derselbe ein Enzym absondert, welches auf das Rindengewebe der Mutterwurzel lösend einwirkt und so der jungen Seitenwurzel den Weg nach aussen bahnt. In seinem „Traité de Botanique“" ) äussert er sich ferner wörtlich in folgender Weise: "Plus tard, un peu avant la sortie, la poche digestive est détachée à la base et la radicelle en emporte avec elle au dehors le bonnet supérieur, qui s'exfolie en une fois s'il est simple, en une ou plusieurs fois s'il est composé, pour mettre bientôt à nu la surface propre, c'est-à-dire l'épiderme de la radicelle“.

Ich hatte Gelegenheit ${ }^{3}$ ), eine ganz hervorragende, mikrotomisch angefertigte Serie über die endogene Entstehung der Seitenwurzeln bei Cucurbita Pepo

1) Recherches comparatives sur l'origine des membres endogènes. Annales des Sciences natur., 1888, Sér. VII, Tom. VIII.

2) Deuxième édit., Paris, 1891, 1e Partie, pag. 711.

3) Die Gelegenheit wurde mir durch die Güte des Herrn Dr. Boнumin NÈmec geboten, wofür ich ihm an dieser Stelle meinen herzlichen Dank ausspreche. 
zu studieren und gelangte dabei zu dem Resultat, dass „la poche digestive“, welche hier sehr deutlich angelegt wird, niemals intakt an die Peripherie der Mutterwurzel gelangt, um erst dort abgestossen zu werden, wie das, wenn die Angabe van Tieghem's richtig wäre, der Fall sein müsste. Dagegen fängt die "poche" schon ziemlich früh an, sich zu desorganisieren und mit ihr gleichzeitig die zunächst aufliegenden Rindenschichten. Diese Desorganisation führt schliesslich zu einer vollständigen Verschleimung der "poche" und der übergelagerten Rindenschichten, und längs dieses Weges schiebt sich nun die junge Seitenwurzel aus dem Inneren der Mutterwurzel nach aussen hervor.

Dass der Schleim an der Wurzelspitze und den Wurzelhaaren, wie Schwarz hervorhebt, auch vorteilhaft als Verbreitungsmedium für die von der Wurzel ausgeschiedene Säure dienen kann, indem auf diese Weise eine Oberflächenvergrösserung für ihre lösende Wirkung auf die umgebenden Erdpartikeln erzielt wird, ist sehr einleuchtend, und dieselbe Wirkung werden bei der endogenen Entwickelung der Seitenwurzeln wohl auch die Schleimbildungen auf die umschliessenden Rindenschichten ausüben. 
Andererseits ist aber hervorzuheben, dass die herauswachsende Seitenwurzel durch den Schleim eine Schmiere erhält, welche die Reibungen von Seiten der zu durchbrechenden Rindenschicht beträchtlich verringert.

Von grosser Bedeutung ist ferner die zuerst von Darwin und später von Schwarz hervorgehobene Funktion der Verklebung. Die innige Verwachsung der Wurzelhaare mit den Bodenteilchen, die durch die hier wie eine Cementschicht zwischen beiden wirkende Schleimschicht $\mathrm{zu}$ Stande kommt, ist selbstverständlich für die Befestigung der Pflanzen im Boden von dem allergrössten Wert.

Die bei den in die Erde eindringeuden Wurzeln allgemein verbreitete Gleiteinrichtung wird ohne Zweifel noch bei anderen Gliedern von Gefässpflanzen, die sich in die Erde einbohren, angetroffen werden. Bei Rhizomen, wo man ihr Vorkommen erwarten sollte, habe ich bis jetzt vergebens danach gesucht. Es mag dies darauf zurückzuführen sein, dass die zarten Vegetationspunkte nicht unmittelbar mit der Erde in Berührung treten, sondern durch Blattgebilde geschützt sind.

Von grossem Interesse für unsere Fragestellung 
sind dagegen die von G. $\mathrm{K}_{\text {ARsten }}{ }^{1}$ ) in Mexiko an der strandbewohnenden Nyctaginee Okenia hypogaea gemachten Beobachtungen.

Diese Pflanze ist ausgezeichnet durch weithinkriechenden, auf dem Sande niederliegenden Stamm, an dessen Blüten, unmittelbar nach dem Abblühen, sich eine Abwärtskrümmung des Stiels bemerkbar macht. Unter starker Verlängerung gräbt sich der Letztere bis über einen Fuss tief in den lockeren Sandboden ein. Durch eigentümliche Vorgånge, betreffs welcher auf die Schrift selbst zu verweisen ist, wird ein „ausserordentlich zweckdienlicher Apparat geschaffen, der beim Eindringen in den losen Sand möglichst wenig Widerstand bietet: eine Kegelspitze und eine rings glatte, feste Aussenmembran dahinter. Die Aehnlichkeit mit einer Wurzelspitze ist in's Auge fallend. Bei der Reibung an den Erd- und Sandpartikelchen werden die Aussenreihen der verquollenen Zellen an der conischen Spitze verbraucht, yenau so wie die Aussenreihen der Wurzelhaube.... So ist auch hier wieder zu constatieren, wie ganz verschiedene Organe durch

1) Notizen über einige mexikanische Pflanzen. Berichte der Deutschen Bot. Gesellsch., 1897, pag. 14. 
die gleichen äusseren Verhältnisse, denen sie zu genügen haben, gezwungen werden, eine weitgehende äussere Aehnlichkeit anzunehmen". Wir fügen hinzu, das die Karsten'schen Beobachtungen an einem Pflanzenteil, wo von Schutz gegen Vertrocknen, oder anderen Verrichtungen, die man dem Schleim an Wurzelspitzen zugeschrieben hat, keine Rede sein kann, in unwiderlegbarer Weise für die Gleitfunktion sprechen.

B. SCHLIIMBLDUNG AN DEN IM INNEREN DER WIRTHSPFLANZEN WACHSENDEN ORGANEN PARASITISCHER GEWAECHSE.

Betrachten wir zuerst die Wachstumsverhältnisse der "Rindenwurzeln" von Viscum album. Ihr anatomischer Bau ist, wie bekannt, von demjenigen ächter Wurzeln sehr abweichend; sie bestehen aus einem kleineren oder grösseren Bündel von Gefässen, welches von einer dünneren Schicht langgestreckter, protoplasmareicher Elemente umhüllt wird.

Graf Hermann zu Solms-Laubach, der in einer Arbeit: „Ueber den Bau und die Entwickelung der 
Ernährungsorgane parasitischer Phanerogamen " ${ }^{1}$ ) auch Verlauf und Bau dieser Rindenwurzeln eingehend untersucht hat, spricht sich auf Seite 607 folgendermaassen aus: „Schreitet man mit der Freilegung einer Rindenwurzel vorsichtig gegen die Spitze hin fort, so sieht man sie langsam, aber stetig schmächtiger und dünner werden, wobei zugleich ihr schönes Grün allmählig in immer blassere und gelblichere Färbung übergeht. Zuletzt endigt sie mit einem sehr schlanken, blassgelblichen, halbdurchscheinenden Kegel, der ausser jedem Verband mit dem umgebenden Gewebe der Nährrinde steht und sich mittelst der Nadel leicht aus demselben herausheben lässt, wobei er sich als von einer wasserhellen, schleimigen, bisweilen fadenziehenden Substanz umgeben erweist. Die gesammte Wurzelspitze besteht aus einer Parenchymmasse, wovon alle oberflächlichen Zellen seitlich aus ihrem Zusammenhange gelöst erscheinen und zu stumpfkeuligen, haarartigen Gebilden von beträchtlicher Länge ausgewachsen sind, sodass dadurch die ganze Wurzelspitze den Anschein eines mehr oder minder wurzelmässigen Pinsels erhält“.

1) In Pringsheim's Jahrbüch., 1868, Bd. 6, pag. 509—633. 'Taf. XXXII-XXXIX. 
Ein ganz ähnliches und hiermit sehr gut zu vergleichendes Verhalten zeigen auch die Wachstumsverhältnisse der Rhizomorphen, wie z. B. Rhizomorpha subcorticalis, welche parasitisch vorzugsweise auf Nadelhölzern (Kiefern), auch saprophytisch auf toten Stämmen vieler Laubhölzer sehr verbreitet vorkommt.

Untersucht man die äusserste Spitze einer solchen Rlizomorpha, so sieht man, dass dieselbe aus zartwandigen, plasmatischen, untereinander nicht verwachsenen, sondern parallel nebeneinander laufenden Hyphen besteht, die aber keineswegs von gleicher Länge sind, von denen vielmehr manche den anderen etwas vorausgeeilt. Die Wandung dieser einzelnen Hyphen ist wenig scharf konturiert und gleichsam in eine gallertartige Grundmasse eingebettet, welche den plasmatischen Inhalt der einzelnen Hyphen von einander trennt.

Ebenso wie die einzelnen Hyphen solch einer Rhizomorphen-Spitze verhalten sich die haarartigen Gebilde einer Rindenwurzelspitze von Viscum album. Weder Graf Solms, noch BREFeld '), noch HaR-

1) Botan. Untersuch. über Schimmelpilze, Leipzig, 1877, Heft III, pag. 136-173. Taf. X-XI. 
TIG ${ }^{1}$ ) geben uns eine Andeutung über die Funktion des Schleimes in diesen beiden Fällen; sie erwähnen sein Vorkommen, ohne auf seine Bedeutung einzugehen.

Ueber die Schnelligkeit, mit welcher das Spitzenwachstum der Rhizomorphen-Stränge vor sich geht, sind wir durch die Arbeiten von BReFeld und Hartig unterrichtet.

BREFELd 2) sagt darüber: „Der Act des Eindringens dauerte etwa 1 bis 2 Tage, in den weiteren Tagen erfolgt das rapide Wachstum des eingedrungenen Stranges in der cambialen Region der Wurzel, von dieser reicher ernährt, so dass in der kurzen Frist von 6-8 Tagen eine Strecke von 5-6 Zoll von dem Pilze im lebenden Holze durchwachsen wird".

Und Hartig ${ }^{3}$ ): „In einem Zeitraume von 4 Wochen war der Pilz auf $1 \mathrm{~cm}$. Tiefe eingedrungen und zwar nicht nur in den Gefässen, die völlig von Mycel ausgefüllt waren, sondern auch in den anderen Elementen des Holzkörpers. An einer

1) Untersuch. aus dem forstbotan. Instit. zu München, Berlin, 1883, Heft III, pag. 95-140. Taf. VI-VII.

2) L. c., pag. 151.

3) L. c., pag. 107. Taf. VII, Fig. 36 und 37. 
Ahornwurzel, welche sich grösstenteils im Wasser befand, war das Mycel in derselben Zeit $2 \mathrm{~cm}$. tief in Gestalt üppiger Rhizomorphen im Rindengewebe eingedrungen“.

Bei diesem raschen Vorwärtsdringen müssen nicht unbeträchtliche Hindernisse überwältigt werden. Wenn auch bei der Ueberwindung derselben chemische Auflösungsprocesse eine beträchtliche Rolle spielen mögen, so gehen wir doch sicher nicht fehl, wenn wir dem Schleim der Rlizomorphen, wie auch der Rindenwurzeln von Viscum, dieselbe Bedeutung zuschreiben, wie demjenigen der Wurzelspitzen: die Reibungswiderstände werden durch den als Schmiere wirkenden Schleim vermindert, und nebenbei wird auch den zarten Spitzen ein Schutz gewährt gegen mechanische Verletzungen. 


\section{ABSCHNI'YT.}

\section{Schleimbildung bei den Bryophyten.}

SCHLEIMBILDUNG BEI DEN HEPATICAE.

Obwohl das Vorkommen einer oberflächlichen Schleimbildung an den Vegetationspunkten von Lebermoosen von älteren Forschern wiederholt beschrieben wurde, so ist doch LeitgeB ') derjenige, bei dem wir die ersten genauen Angaben über die Quelle dieser Schleimbildung finden. In der letzten Zeit ist es in erster Linie GoebeL ${ }^{2}$ ) gewesen, der diesem Thema in mehreren Arbeiten sein Interesse zugewandt hat, und nach ihm Roge ${ }^{3}$ ),

1) Untersuchungen über die Lebermoose, 1874-1881.

2) Archegoniaten-Studien. Flora, 1892, Ergänzungsb., und 1893.

Morphologische und biologische Studien. Annales du Jardin bot. de Buitenzorg, Vol. VII. IX.

3) Beiträge zur Kenntniss der Veyetationsorgane der Lebermoose. Flora, 1893, pag. 279-312. 'Taf. IV. 
der die Vegetationspunkte der Lebermoose speciell auf die dort vorkommende Schleimbildung eingehender untersuchte.

Was die Verbreitung solcher Schleimbildungen betrifft, so genügt es, wenn ich die Aeusserungen der beiden letztgenannten Forscher darüber hier wiedergebe.

Goebel ") sagt: „Der Vegetationspunkt vielleicht aller Lebermoose ist mit Schleim bedeckt, ebenso vielfach die Geschlechtsorgane“, und RuGE ${ }^{2}$ ): „Eine Absonderung von Schleim erfolgt wohl bei allen Lebermoosen ohne Ausnahme“.

Da es nicht meine Absicht ist, die Zahl der bekannten Beispiele zu vermehren, so wende ich mich gleich zur Erörterung der Funktion dieser Exkrete. Die Bedeutung, welche Goebel dem Schleim bei den Lebermoosen zuschreibt, liegt wiederum zunächst in dem Schutz gegen Austrocknung, obwohl zuweilen auch hier die Fähigkeit des Schleimes in Betracht kommen soll, das Wasser festzuhalten und auf diese Weise seinen Hindurchtritt in das Innere der Pflanze zu erschweren. Ruge als Schűler Goebel's

1) Pflanzenbiolog. Schilderungen, II, pag. 209.

2) L. c., pag. 295. 
schliesst sich vollkommen den Ansichten seines Lehrers an.

Der Sitz der Schleimbildung ist bei allen Lebermoosen auf das Engste an die im Wachstum begriffenen Teile geknüpft. Der Vegetationspunkt ist von einer mehr oder weniger mächtigen Schleimschicht umhüllt, welche entweder oberflächlichen Trichomen (Marchantiales und Jungermaniales), oderinneren Schleimhöhlen (Anthocerotaceae) ${ }^{1}$ ) entstammt.

Bei den Marchantiales sind es die Schuppenblätter oder Ventralschuppen, welche im jüngsten Zustande die Funktion der Schleimbildung ausüben. Die erste Anlage der Ventralschuppe geht, wie schon НоғмєsTER ${ }^{2}$ ) angiebt, in der Weise vor sich, dass eine unmittelbar hinter dem Scheitelrande gelegene Zelle zu einer Papille auswächst, die sich durch eine Querwand vom ursprünglichen Zellraume abgrenzt. Diese Papille füllt sich später mit Schleim und ergiesst beim Platzen denselben über den Vegetationspunkt. Die Zerreissung besagter Schleimpapille findet meistens frühzeitig statt, woher es kommt, dass diese

1) Ich folge hier der Einteilung von Schiffner. Siehe Engter und Prantl, Natürl. Pflanzenfamilien, 1893-1895, Th. I, Abth. 3, Bogen 1-9, Lief. 91. 92. 112.

2) Vergl. Untersuch. höherer Kryptogamen, 1851, pag. 52. Taf. XIl, 14l, bei $a$. 
auch an verhältnissmässig jungen Schuppen oft nicht mehr aufzufinden ist.

Von den Ricciaceen untersuchte ich selbst Riccia glauca, bei welcher die Ventralschuppen nur schwach entwickelt sind, von der ich aber erwähnen will, dass die papillenförmige Epidermis den Eindruck macht, als ob sie selbst auch die Fähigkeit einer diffusen Schleimabsonderung besitze; denn die Schleimschicht markiert sich hier so zu sagen von selbst dadurch, dass allerlei kleine Körperchen (vor Allem Bakterien, kleine einzellige Algen, Diatomeen u. s. w.) an ihrer Oberfläche haften.

Bei den Jungermaniales finden wir die charakteristischen Amphigastrien (Jungerm. acrogynae) oder Amphigastrien ähnliche Gebilde (Jungerm. anacrogynae), welche im jugendlichen Zustande hauptsächlich die Schleimbildung vermitteln und den Vegetationspunkt mit einer kontinuierlichen Schleimschicht umhüllen. Sie entwickeln sich aus der bauchständigen Zelle, die durch jede erste Teilungswand jedes Segmentes, welche nach der Unterseite geneigt ist, abgeschnitten wird. Diese jugendlichen Amphigastrien, welche LeıтgeB "Primordialpapillen“ nennt, krümmen sich nun mit ihrem vorderen Ende nach aufwärts und ungeben auf diese Weise den 
Vegetationspunkt. Leitgeb ${ }^{1}$ ) sah in diesen Primordialpapillen eine Stütze seiner Auffassung, dass ursprünglich bei allen beblätterten Jungermaniaceen noch eine dritte Blattreihe vorhanden war, dass diese aber später verkümmerte und nur die Primordialpapillen von ihr erhalten blieben.

Was die Allgemeinheit des Auftretens dieser Primordialpapillen bei den foliosen Jungermaniaceen betrifft, so sagt Leir'ieb darüber Folgendes ${ }^{2}$ ): „Es kommt nur höchst selten vor, dass die bauchständigen Segmente nicht schon bald nach ihrer Anlage typische Anhangsgebilde produciren. Ich kenne in dieser Beziehung nur Radula und Lejeunia calcarea. Bei allen übrigen Jungermaniaceen aber, auch jenen, welchen nach den Angaben der Schriftsteller selbst rudimentäre Amphigastria durchaus fehlen, finden wir, dass jedes ventrale Segment bald nach seiner Anlage ein Anhangsgebilde producirt, das in seiner einfachsten Form als eine ein- oder zweizellige keulenförmige Papille erscheint".

Diese Primordialpapillen verschwinden übrigens verhältnissmässig früh; selten sind sie noch an ganz ausgebildeten Amphigastrien anzutreffen.

1) L. c., 1875, Heft II, pag. 16.

2) L. c., 1875, Heft Il, pag. 7. 
Bei den frondosen Jungermaniaceen (ebenso bei den Marchantiaceen) ist die Schleimpapillenbildung nicht nur auf die ventrale Seite beschränkt, sondern auch an der Dorsalseite des Thallus tritt sie hier auf, aber immer in geringerer Zahl.

Bei der dritten grossen Gruppe der Hepaticae den Anthocerotaceen - findet nun ebenfalls eine Schleimabsonderung statt; hier ist es jedoch keine trichomatische, sondern eine innere Schleimbildung. Die sogenannten Schleimspalten entstehen auf der Unterseite des Thallus, dicht hinter dem fortwachsenden Scheitel, und ihre Funktion besteht in der Absonderung des Schleimes, womit die Intercellularräume unter ihnen gefüllt sind. In Folge dessen ist der fortwachsende Vegetationspunkt immer in derselben Weise, wie bei den vorher besprochenen Gruppen der Lebermoose, mit einer Schleimschicht überzogen.

Wie bekannt, erfolgt dann weiterhin die Infektion durch Nostoc-Kolonien (Notoc lichenoides), und in Folge dessen eine raschere Teilung der umliegenden Zellen, deren Resultat der Schluss des Schleimspaltes ist. Der Schleimspalt fungiert hier jeweilig nur so lange, wie er in unmittelbarer Nähe des Vegetationspunktes liegt; ist dieser aber weiter gewachsen, so 
kann er entbehrt werden, weil im jüngsten Teil schon ein neuer Schleimspalt sich entwickelt hat, der seine Funktion übernimmt.

Vergleichen wir jetzt die Schleimbildung dieser drei verschiedenen Gruppen der Hepaticae mit eineinander, so kommen wir zu dem Resultat, dass dieselbe bei allen in zwei Punkten übereinstimmt, nämlich: erstens darin, dass sie bei den Lebermoosen eine vorübergehende ist, und zweitens darin, dass sie auf das Engste an die jüngsten Entwickelungsstadien des Wachstums geknüpft ist.

In dem Versuch, der Schleimbildung eine biologische Deutung zu geben, ist für Goebel auch der jeweilige Feuchtigkeitsgehalt des Standorts maassgebend. Dem ist aber entgegenzuhalten, dass die Mehrzahl der Lebermoose an feuchten, schattigen Standorten lebt (feuchten Stämmen, Erde, Steinen u. s. w.), und mithin die Notwendigkeit specieller Anpassungseinrichtungen gegen Vertrocknung nicht recht einzusehen ist.

Ueber die andere Rolle, die Goeben dem Schleim zuschreibt, nämlich den Hindurchtritt von Wasser zu erschweren, gehe ich an dieser Stelle hinweg, da ich später ausführlich darlegen werde, dass diese 
Auffassung einer genaueren Prüfung gegenüber unhaltbar ist.

Für unseren Standpunkt in der Deutung der Schleimfunktion sind nicht die Standortseigentümlichkeiten maassgebend, sondern die specifischen Wachstumsverhältnisse der Lebermoose, ihr dem Substrat angeschmiegter, kriechender Wuchs, wie er allen gemeinsam ist, mit Ausnahme z. B. von Haplomitrium Ilookeri und Calobryum Blumei, welche in Form eines beblätterten Stengels aufrecht wachsen, und von Riella, der einzigen unter Wasser lebenden Gattung.

Bei den kriechenden, dem Substrat angeschmiegten Lebermoosen ist es nämlich unvermeidlich, dass beim Fortwachsen des Thallus (Narchantiales, frondose Jungermaniaceen und Anthocerotaceen), oder der jüngeren Stengelteile (foliose Jungermaniaceen), Reibungen stattfinden müssen zwischen den im Wachstum begriffenen Teilen und dem Substrat, dem sie angeschmiegt sind, und zwar sind es die zarten Sprossspitzen, welche diesen Reibungen fast ausschliesslich unterworfen sind; und gerade an diesen Stellen findet man immer eine ausgeprägte Schleimbildung, sodass es ganz zweifellos ist, dass der Schleim hier beim Fortwachsen eine wichtige 
Rolle als Schmiere spielt, um erstens das Fortkriechen über das Substrat zu erleichtern, zweitens einen Schutz zu gewähren gegen mechanische Verletzungen, welchen der Thallus auf seinem Wege ausgesetzt sein kann.

Zur Stütze dieser Auffassung will ich einige wenige Beispiele anführen. Wo wir bei den Thallus-Lebermoosen eine ausgeprägte, deutlich nach der Ventralseite hervortretende Mittelrippe finden, wie z. B. bei Blyttia, Moerkia, Umbraculum, Podomitrium u. s.w., da können wir konstatieren, dass die Anordnung der Schleimpapillen hauptsächlich, ja selbst oft ausschliesslich (Blyttia), auf die fortwachsende Mittelrippe beschränkt ist.

Ein ähnliches Verhalten finden wir bei Treubia insignis. Gorbed entdeckte dieses Lebermoos im Jahre 1886 bei Tjibodas (Java) und beschrieb es zum ersten Mal in den "Annales du Jardin bot. de Buitenzorg" von 1891 '). Durch die Güte des Herrn Prof. Strahl, welcher mir das von ihm auf Java gesammelte Material überliess, wurde mir die Gelegenheit gegeben, dieses interessante Lebermoos zu untersuchen.

Der ausgezeichneten Beschreibung GorbeL's habe

1) Vol. IX, pag. 1-40. 
ich nichts hinzuzufügen; nur möchte ich hier kurz die Lokalisation des Schleimes hervorheben, dessen Anordnung deutlich für die hier vertretene Auffassung spricht.

Treubia insignis hat an ihrer Ventralseite keine Spur von Anhangsgebilden; ebenso fehlen hier auf der Unterseite des Stämmchens die Schleimpapillen; dagegen besitzt jedes Blatt an seinem unteren Rande einen Flügel (eine Wucherung des Blattrandes), welcher besonders reichlich mit schleimabsondernden Papillen besetzt ist. Dieser ventrale Blattflügel übernimmt also die Schleimbildung, und nach seiner Lage dem Substrat gegenüber muss auch hier wieder geschlossen werden, dass seine Schleimsekretion dazu dient, die Reibung beim Fortwachsen zu vermindern. Genau dasselbe finden wir bei der amphigastrienlosen Gattung Radula und bei Lejeunia calcarea; hier sind es die Seitenblätter (Unterlappen), welche ganz gleich sich verhaltende Schleimpapillen für denselben Zweck entwickeln. 


\section{ABSCHNITT.}

\section{Schleimbildung bei den Pteridophyten.}

SCHLEIMBILDUNG BEI DEN FILICES.

Die oberflächliche Schleimbildung bei den Farnen ist bisher nur selten etwas eingehender untersucht worden.

KÜнN ${ }^{1}$ ) macht einige kurze Bemerkungen über diesen Gegenstand. Die wesentlichsten Angaben verdanken wir Goebeler ${ }^{2}$ ), dessen Arbeit jedoch, trotzdem sie in mehreren Beziehungen Wertvolles bietet, keine Berücksichtigung gefunden hat.

KÜнN ${ }^{3}$ ) sagt vom Schleim bei Farnen, dass er „als Schutz des jugendlichen Blattes gegen Austrocknen" wirksam sei, stimmt also ganz mit der schon mehr-

1) Untersuchungen über die Anatomie der Marattiaceen und anderer Gefässkryptogamen. Flora, 1889, pag. 457-504. Taf. XVIII-XX.

2) Die Schutzvorrichtungen am Stammscheitel der Farne. Flora, 1886, n. 29-31. Taf. XI.

3) L. c., pag. 502. 
fach erwähnten Goebel'schen Ansicht überein. GoeBELER ${ }^{1}$ ) sieht die Bedeutung des Schleimes „in dem Schutz gegen Mangel an Wasserzufuhr und übermässige Erhöhung der Transpiration".

Inwiefern unsere Auffassung von diesen beiden abweicht, wollen wir jetzt darzulegen suchen.

Die Blätter der Farne zeigen in der Knospenlage ein verschiedenes Verhalten, welches oft typisch ist für bestimmte Abteilungen. Den Arten, bei denen das Blatt in der Knospenlage schneckenförmig eingerollt ist, stehen andere gegenüber, bei welchen dies nicht der Fall, ein Umstand, den Ascherson ${ }^{2}$ ) benutzt hat, um die Pteridophyten in Planithallosae und Tuberithallosae einzuteilen. Weiter findet man als charakteristische Merkmale entweder eine dichte, oder leichte Bekleidung mit Spreuschuppen, oder selbst ein völliges Fehlen derselben.

Die Bedeutung dieser Spreuschuppen ist wohl nicht anders aufzufassen denn als Schutzeinrichtung, einerseits gegen die Unbill des Klimas, andererseits als Schutz gegen Tierfrass und Fäulniss. Für das Erstere spricht der Umstand, dass bei den Arten,

1) I. c., pag. 403.

2) Synopsis der mitteleurop. Flora, Leipzig, 1896, Bd. I, Lief. I, pag. 4. 
die in Europa eine mächtige Spreuschuppenbekleidung besitzen, dieselbe in den Tropen in bedeutend geringerem Maasse vorhanden ist; die zweite Schutzwirkung wird namentlich durch die reichliche Imprägnierung der Spreuschuppen mit Gerbstoff bedingt.

An den Spreuschuppen treten, sowohl am Rande, als auch an der Spitze, häufig Schleimdrüsen auf. Die hauptsächliche Schleimbildung aber, welche ich hier ausführlicher besprechen will, geht aus von haarförmigen Trichomen, die in geringer Zahl an der Oberfläche des eingerollten Wedels, dagegen mehr nach innen $\mathrm{zu}$ in grosser Menge auftreten. Diese haarförmigen Trichome haben eine angeschwollere Endzelle, welche mit Schleim gefüllt ist und bei Wasserzufuhr platzt.

Die Stellen, wo diese Schleimhaare sich am Reichlichsten finden, liegen immer an der Rücken- und Bauchseite des eingerollten Wedels. Mehr nach innen zu treten sie am Häufigsten dort auf, wo die Ränder des eingerollten Blattstiels mit einander in Berührung treten.

Goebeler sagt über Osmunda regalis Folgendes: „Dagegen auf dem Rücken und Bauch der Wedel und auf den Flügeln der Blattstiele, also überall, 
wo die Ränder eines Wedels und seiner Blattstielflügel sich auf die jungen Knospenteile auflegen, stehen dicht gedrängt und verflochten die hygroskopischen Rosenkranzhaare, wovon die oberen, schleimhaltigen Zellen beim Zutritt von Wasser platzen", u.s.w. Er hebt also schon hervor, dass überall, wo in der Knospe Berührung von aufeinandergelagerten Teilen stattfindet, auch diese Schleimhaare auftreten.

Meine eigenen Untersuchungen haben mich weiter gelehrt, dass diejenigen Farnwedel, welche am Festesten eingerollt sind, die reichlichste Anhäufung von Schleimhaaren zwischen ihren Windungen nachweisen lassen, dass dagegen bei den locker oder gar nicht eingerollten Wedeln diese Schleimbildung ganz unterbleiben kann. Es existiert also eine Beziehung zwischen der Beschaffenheit des Wedels und der dort auftretenden oberflächlichen Schleimbildung. Weiter ist auffallend, dass da, wo die Schleimbildung am Stärksten auftritt, die Spreuschuppenbildung ganz und gar unterbleibt und jene also deren Funktion zu übernehmen scheint.

Die Wedel der Asplenien, von denen ich $A$. bulbiferum, A. dimorphum, A. Belangeri, A. Patersoni, A. mexicanum, A. Felix femina, A. Goeringianum, A. Adi- 
antum nigrum, $A$. Ruta muraria, A. crenatum, A. Serpentini, A. Nidus, A. heterodon, A. maximum untersucht habe, sind alle ziemlich fest eingerollt, und die Schleimhaare fehlen denn auch niemals zwischen den Windungen. Weitere schöne Beispiele, bei denen diese Schleimhaare reichlich gefunden werden, sind: Onychium japonicum, Trichomanes radicans, Blechnum Spicant, Aspidium Serra, Polypodium pustulatum u. s. w.; aber keine Form ist in dieser Hinsicht zu vergleichen mit dem, in feuchten Waldschluchten West-Java's, an Bächen auftretenden Nephrodium callosum.

$\mathrm{Da}$ in dem hiesigen botanischen Garten lebende Pflanzen dieser Art sich finden, welche Prof. Stahl von seiner Reise auf Java mitgebracht hat, so war mir die Möglichkeit gegeben, die Schleimbildung an frischem Material genau zu verfolgen und einige nachher zu erwähnende Versuche anzustellen.

Von Farnen, bei denen die Schleimbildung niemals auftritt, erwähne ich die Adiantum-Arten, Ophioglossum und Botrychium. Bei der ersten Gattung finden sich meist locker eingerollte Wedel, bei den beiden anderen ist eine schneckenförmige Einrollung überhaupt nicht vorhanden.

Wenn oberflächliche Schleimbildung wirklich dazu 
dienen sollte, das jugendliche Blatt gegen Austrocknen und übermässige Transpiration zu schützen, wie KüHN und Goebeler es annehmen, dann sollte man erwarten, dass gerade bei Adiantum dieselbe von grossem Nutzen für das junge Blatt sein müsste.

Diese Voraussetzung trifft jedoch keineswegs zu. Legt man nämlich an einem Adiantum-Wedel die jungen Fiedern vorsichtig auseinander, so sieht man, dass auch die jüngsten, zartesten Blattzipfelchen, welche sich noch im embryonalen Zustande befinden, frei bis an die Peripherie zu beiden Seiten des Wedels ragen, ohne dass sich hier die geringste, äusserlich erkennbare Einrichtung finden liesse, welche im Stande wäre, dieselben gegen schädliche äussere Einflüsse zu schützen. Hier fehlen nämlich die Spreuschuppen vollständig, oder dieselben sind nur in sehr geringer Zahl vorhanden, und eine Schleimabsonderung fehlt immer gänzlich.

Goebeler's Auffassung über die Funktion des Schleimes veranlasst ihn weiter, eine Hypothese aufzustellen über die specielle Anpassung der Farne an ihre Standorte. Er sagt ${ }^{1}$ ): „Farne, welche einen

1) L. c., pag. 491 . 
kontinuierlich feuchten Standort lieben, bedürfen einer solchen Anpassung (nämlich einer Schleimbildung) nicht". Dass dies ganz bestimmt nicht richtig ist, könnte man an vielen Beispielen beweisen, so u. A. an Osmunda regalis, welche doch eine reichliche Schleimbildung besitzt und bei der schon die blosse Erwähnung ihres früheren Namens, Osmundd palustris Sturm '), genügt, um zu zeigen, wie wenig mit der Standortseigentümlichkeit die GozbeLER'sche Anschauung übereinstimmt.

Ein weiteres Beispiel, das wohl in dieser Hinsicht durchschlagend ist, findet sich in dem schon erwähnten Neplirodium callosum Bl., bei dem die Schleimbildung der Farne wohl ibr Maximum erreicht hat und welches nach $\mathrm{R}_{\mathrm{ACI}}$ BORSKI ${ }^{2}$ ) immer an "schattigen und feuchten Stellen des Hügellandes und der unteren Waldzone“ Java's wächst.

Ich verzichte darauf, weiter zu prüfen, ob die Schleimbildung in der Richtung, die KüHN und Goebeler angegeben, unter anderen Umstånden vielleicht doch von Nutzen sein kann, und gehe zu der Darlegung meiner Auffassung über. Dass

1) Ноокев, Synopsis Filicum, 1863, pag. 427.

2) Die Pteridophyten der Flora von Buitenzorg. Ie Partie de la Flore de Buitenzorg, Leide, 1898, pag. 192. 
bei der Entfaltung eines fest eingerollten Wedels notwendig Reibungen auftreten, ist selbstverständlich, und gerade an jenen Stellen, wo die Reibungen am Stärksten sind, d. i. an der Rücken- und Bauchseite der Windungen, sehen wir eine massenhafte Anhäufung von S'chleimhaaren. Durch das Sprengen dieser Schleimköpfe werden die sich berührenden und dicht aufeinanderliegenden Teile schlüpfrig gemacht, wodurch ein gegenseitiges Vorübergleiten ermöglicht wird, ein Umstand, der die Entfaltung in nicht geringem Maasse erleichtern muss. Ist die Entfaltungsperiode vorüber, so ist der Zweck dieser Schleimbildung erfüllt, und wir sehen denn auch, dass die Schleimhaare bald darauf vertrocknen und ganz verschwinden.

Für diese Auffassung spricht noch der Umstand, dass da, wo von Reibungen bei der Entfaltung nicht die Rede sein kann, auch keine Schleimbildung zu finden ist. Wir sahen, dass bei Adiantum, wo der Wedel so locker eingerollt ist, dass selbst im jüngsten Zustande eine gegenseitige engere Berübrung der Wedelwindungen beinahe nicht auftritt, wo also bei der Entfaltung keine Reibungen entstehen und auf eine Verminderung derselben hinzielende Einrichtungen überflüssig sind, auch keine 
Schleimproduktion auftritt. Dasselbe ist der Fall bei Ophioglossum und Botrychium, wo eine schneckenförmig eingerollte Wedellage überhaupt nicht vorkommt und die Knospe so locker ist, dass auch da nicht an störende Reibungen gedacht werden kann.

Wir haben oben die Fälle besprochen, wo die Windungen der fest eingerollten Wedel einander eng berühren und dementsprechend eine ausgeprägte Schleimbildung auftritt, welche bei Nephrodium callosum Bl. ihr Maximum erreicht. Hand in Hand mit der in den Vordergrund tretenden Schleimbildung geht hier eine Reduktion der Spreuschuppenbildung, die ebenfalls bei Nephrodium, wo sie gleich Null wird, ihr Minimum erreicht. Im Gegensatz dazu stehen diejenigen Farne, welche eine ganz lockere Wedellage und demgemäss eine Reduktion der Schleimbildung zeigen, die bei Adiantum, Opliioglossum und Botrychium gänzlich unterbleibt. Zwischen diesen beiden Extremen bewegt sich die weitaus grösste Mehrzahl der übrigen Farne, bei denen sich alle möglichen Uebergangsformen nach beiden Richtungen antreffen lassen. Einige Beispiele dafür sind: Dennstaedtia tenera, Pteris allosora, P. cretica, Polypodium vulgare, P. Phyllitidis, Pteris aquilina, Scolopendrium officinarum, Onoclea 
spectabilis, O. sensibilis, mehrere Aspidium-Arten, Woodsia incisa, Cystopteris fragilis, C. bulbifera, Struthiopteris germanica u. s. w.

Bei allen diesen Arten findet sich ebenfalls Schleimbildung, entweder nur an der Spitze und an den Randzacken der Spreuschuppen, oder unter der speciellen Form von Schleimhaaren. In denjenigen Fällen, wo die Schleimbildung gegenüber der mächtigen Spreuschuppenbildung (Aspidien) zurücktritt, mag die Bekleidung mit allerdings trocknen, aber glatten Spreuschuppen ebenfalls dazu dienen, bei der Entfaltung des Wedels das Uebereinanderweggleiten der sich aufrollenden Fiedern zu begünstigen.

Welch eminente Bedeutung auch bei Farnen dem Schleim als Schutzmittel gegen Tierfrass zukommt, mögen folgende Versuche illustrieren, welche neue Belege für die Stahl'sche Ansicht beibringen und deutlich zeigen, dass dem Schleim in dieser Hinsicht nicht bloss eine "untergeordnete Bedeutung“ zukommt, wie dies von Goeber und seinen Schülern angenommen wird.

Ein junger Wedel von Nephrodium callosum $B l$. wurde in fünf Stücke zerlegt. Um das Anfressen der Schnittflächen zu verhindern, wurden dieselben mit Wachs verklebt. Nachdem der Schleim in Wasser 
zum Aufquellen gebracht war, wurden die Stücke in eine Krystallisierschale mit zehn Exemplaren der gefrässigen Limax agrestis gelegt.

Vorber hatte ich am Rücken des Wedels an einer Stelle den Schleim vorsichtig mit Filtrierpapier abgerieben und ebenso bei zwei Stielstückchen die Hälfte der ganzen Oberfläche vom Schleim befreit.

Am anderen Morgen war Folgendes zu konstatieren: Nur diejenigen Stellen, von denen ich den Schleim fortgenommen hatte, waren angefressen worden, sowohl die nur $6 \mathrm{~cm}$. lange schleimfreie Stelle am Wedelrücken, als auch diejenigen Hälften der Stielchen, welche ebenfalls abgerieben worden waren. Jetzt wurde von zwei weiteren Stielstückchen jedesmal die Hälfte vom Schleim befreit. Am folgenden Tage waren wiederum bloss diese Stellen tief angefressen, die übrigen schleimbedeckten Teile völlig unberührt.

Rekapitulieren wir zum Schluss das über die Bedeutung des Schleimes bei F'arnen Gesagte, so kommen wir zu dem Ergebniss, dass die Ansichten von Goebeler und KühN unter gewissen Umständen wohl zu Recht bestehen mögen, indem der Schleim die ihm von diesen Autoren zugeschriebene Bedeutung als Schutzmittel gegen Wasserverlust haben 


\section{4}

mag, dass aber vor Allem in Betracht kommt seine Wirksamkeit als Schmiere, welche die bei der Entfaltung der Wedel entstehenden Reibungen verringert. Hierdurch wird nicht nur der Entfaltungsprocess erleichtert, sondern zugleich auch die Gefahr von Verletzungen verringert. Nicht zu unterschätzen ist endlich die Rolle des Schleimes als Schutzmittel gegen Tierfrass, in solchen Fällen wenigstens, wo er die Stelle der fehlenden oder doch stark zurücktretenden Spreuschuppen vertritt. 


\section{ABSCHNITT.}

\section{Schleimbildung bei den Laubknospen.}

Wie schon in der Einleitung bemerkt worden ist, war es Hanstein, welcher im Jahre $186 \mathrm{~S}$ zum ersten Mal die oberflächliche Schleimbildung eingehend untersucht und als Untersuchungsmaterial die Laubknospen benutzt hat. Er kam zu dem Resultat, dass die Bekleidung mit Schleim eine schützende Wirkung ausübe zur Verminderung der Ausdünstung, zur Erhöhung der Turgescenz und somit zur Begünstigung der Entwickelung der Knospenteile.

Die Pflanzen, bei denen Hanstuin die oberflächliche Schleimbildung am Stärksten ausgeprägt fand, waren die Polygoneen, z. B. Polygonum, Rumex und 
Rheum. Die Quelle dieser Schleimbildung fand er vor Allem in den inneren Flächen der häutigen Verlängerungen der Blattscheiden (Ochreae), in welchen die jüngeren Knospenteile während ihres Heranwachsens immer eng und lange eingehüllt bleiben.

Im Inneren dieser Ochreae finden sich halb haar-, halb lappenförmige Anhängsel (Colleteren) in grosser Zahl, und diese Gebilde liefern den Schleim, der es bedingt, dass eine solche Knospe vollkommen schlüpfrig anzufühlen ist. Auch die Blattstiele sind immer damit bedeckt, und zwar soweit sie mit jenen Scheiden verwachsen sind.

Warum nun gerade die Polygoneen eine so reichliche Schleimbildung führen und auf welche Weise sie dieselbe verwenden, werde ich jetzt klarzulegen versuchen.

Für diesen Zweck ist es vielleicht gut, vorher eine Anzahl von Beispielen aus anderen Familien $\mathrm{zu}$ nennen, bei denen es mir gelang, ebenfalls eine oberflächliche Schleimbildung nachzuweisen.

So fand ich dieselbe bei: Ficaria ranunculoides, Ranunculus cassubicus, Helleborus-Arten, Viola sylvestris, $V$ aleriana Phu, Centranthus ruber, Valerianella coronata, $V$. Szovitsiana, $V$. auricula, $V$. eriocarpa, Chenopodium 
bonus Henricus, Saxifraga crassifolia u. s. w., ebenso bei Keimpflänzchen von Isatis und Coreopsis grandiflora.

Alle diese Pflanzen, zu wie verschiedenen Familien sie auch gehören mögen, stimmen in dem einen Punkte überein, dass sie alle mehr oder weniger gut entwickelte Blattscheiden besitzen, in welchen immer die jüngeren Knospenteile während ihres Heranwachsens fest eingehüllt liegen. Scheidenförmige Ausbildung des Blattgrundes und oberflächliche Schleimbildung scheinen also hier in Korrelation zu stehen, sodass wir uns fragen müssen, wie dieser Zusammenhang aufzufassen ist.

Wie schon gesagt, ist in der Knospenlage das junge Blatt immer von der Scheide des nächst älteren Blattes eng eingehüllt, sodass beim Herauswachsen des jungen Blattes aus der umhüllenden Scheide ein gewisser Reibungswiderstand zu überwinden ist. Erleichtert wird dies auch hier durch den als Schmiere wirkenden Schleim.

Aber nicht nur an der Innenseite der Scheiden, sondern auch an den umhüllten jungen Blättern finden sich oft die Schleimhaare. Bei Rumex Patientia, R. alpinus, Polygonum cuspidatum, Rheum-und Chenoportium-Arten, Oxyria elatior, 'Tradescantia Zebrina 
u. s. w., wo die jungen Blätter selber von den Blatträndern aus nach vorn oder rückwärts eingerollt sind, fand ich immer an deren Oberfläche, sowohl an der Unter- als der Oberseite, eine reichliche Schleimpapillenbildung, die ich in demselben Sinne deuten möchte.

B. LAUBKNOSPEN DER MONOCOTYLEDONEN.

Obwohl ich zahlreiche Monocotylen auf das Vorhandensein von Gleitschleim untersucht habe, konnte ich, mit Ausnahme der schon erwähnten Gattung Tradescantia, weder an den Blatt-, noch an den Blütenknospen jemals eine oberflächliche Schleimbildung finden. Man sollte aber gerade hier, wo die Scheidenbildung am Kräftigsten entwickelt ist, im Einklang mit der Auffassung, welche ich im vorigen Abschnitt vertreten habe, eine Schleimbildung erwarten.

Wie das Fehlen derselben zu erklären sein mag, kann ich nur vermutungsweise andeuten. Es kommen hier wohl zunächst in Betracht die viel einfachere Knospenlage und die einfachere Gestalt der Blätter, bei denen Einbuchtungen, 
Zacken u. s. w., welche bei den Dicotylenblättern fast allgemein verbreitet sind, nicht vorkommen, und in Folge dessen ein grosses Hinderniss weniger vorhanden ist. Ausserdem ist vielleicht die hervorragende Glattheit der einander berührenden Teile in Betracht zu ziehen, sowie der Umstand, dass die Epidermis nicht, wie bei den Dicotyledonen, meist aus isodiametrischen, sondern aus langen, schmalen Epidermiszellen besteht. Ferner mag es auch vorteilhaft sein, dass die äusseren Membranen der Epidermiszellen hier bedeutend dicker sind, als bei den Dicotylen. Endlich spielt vielleicht auch die schwere Benetzbarkeit der Monocotylenblätter eine Rolle in dieser Richtung, ähnlich wie bei den eingerollten Blättern von Nelumbium, wo, wie Schiluing ${ }^{1}$ ) gezeigt hat, ebenfalls eine Schleimbildung fehlt.

1) L. c., pag. 310 . 


\section{ABSCHNITT.}

Schleimbildung bei den Wasserpflanzen.

Bei dieser Gruppe von Pflanzen ist oberflächliche Schleimbildung eine ausserordentlich häufige Erscheinung, die wir an den verschiedensten Stellen auftreten sehen. GoebeL ${ }^{1}$ ) bespricht in seinen „Pflanzenbiologischen Schilderungen“, in einem speciellen Abschnitt über Wasserpflanzen, die besonderen Lebensbedingungen derselben und die damit in Zusanmenhang stehenden Struktur-Eigentümlichkeiten. In $\S 2$ jenes Abschnittes geht er sehr ausführlich auf die bei ihnen vorkommende Schleimbildung ein, wobei er seine schon mehrfach erwähnte Auffassung über die Bedeutung des Schleimes als Schutzmittel gegen das Wasser vertritt.

1) L. c., II, pag. 232-237. Taf XXXI. 
Im Jahre 1894 erschien dann eine ganz specielle Arbeit von Schilling ${ }^{1}$ ) über die Schleimbildung der Wasserpflanzen, in der dieser zu demselben Schlusse kommt, wie sein Lehrer. Er drückt seine Ansicht über die biologische Bedeutung des Schleimes in folgenden Worten aus ${ }^{2}$ ): „Der Schleim ist als Schutzmittel der jungen Pflanzenteile gegen die unmittelbare Berührung mit Wasser anzusehen. Er erweist sich für Lösungen gewisser Salze und Farbstoffe im Wasser völlig undurchlässig, so lange er noch nicht in Verquellung begriffen ist. Er wird wahrscheinlich von der Pflanze nur so lange gebildet, bis die Entwickelung des Epidermidalgewebes sowie der Cuticula so weit vorgeschritten ist, bis diese seine Aufgabe übernehmen können. Seine Rolle als Schutzmittel vor Tierfrass und Algenbesiedelung kann nur von untergeordneter Bedeutung sein".

Es sei mir gestattet, die verschiedenen Punkte etwas näher zu prüfen.

Was die beiden ersten betrifft, nämlich einmal den Schutz, welchen der Schleim gegen die

1) Anatomisch-biologische Untersuchungen über die Schleimbildung der Wasserpflanzen. Flora, 1894, Heft 3, pag. 280-360.

2) L. c., pag. 360. 
unmittelbare Berührung der jungen Pflanzenteile mit Wasser bieten soll, und zweitens die Behauptung der Undurchlässigkeit des Schleimes für Lösungen gewisser Salze und Farbstoffe, so werden beide Behauptungen sofort hinfällig durch die Ergebnisse der von Pringsheim angestellten und im Jahre 1895 nach seinem Tode publicierten Untersuchungen: "Ueber chemische Niederschläge in Gallerte“ ${ }^{1}$ ).

Ich unterlasse es, die Resultate dieser Arbeit eingehend $\mathrm{zu}$ besprechen und will hier für meinen Zweck nur folgende Stelle daraus wiedergeben:

„Die Bewegung der Flüssigkeiten in Gallerte ist ja seit Graham bis auf dje neueste Zeit, wenn auch unter Einhaltung anderer Methoden, zur Bestimmung der Diffusionsgrösse benutzt worden, und die fast übereinstimmenden Befunde der Beobachter gestatten wohl die Annahme, dass die in Wasser löslichen Substanzen sich in der Gallerte gerade so bewegen, wie in reinem Wasser" ${ }^{2}$ ).

Die Versuche von Pringsheim wurden alle mit einer 5-procentigen Gelatinelösung angestellt; dagegen gebrauchte GоєвєL für seine Diffusions-

1) Pringsheim's Jahrbüch für wissensch. Botanik, 1895, Bd. 28, Heft 1, pag. 1-38, Taf. XXII.

2) L. c., pag. 33. 
versuche Agar-Gallerte, deren Koncentration er aber nicht angegeben hat. Wenn Goebel dann zu dem Resultate kommt, „dass eine Wasserverschiebung durch osmotische Prozesse in der Gallerte nur langsam vor sich geht", so wird das wohl hauptsächlich dadurch bedingt sein, dass seine Lösungen zu koncentriert gewesen und darum kaum mit der äusserst zarten und wasserhaltigen Schleimbildung, welche wir hier vor uns haben, zu vergleichen sein dürften.

Der folgende Versuch mag zeigen, wie wenig auch ein dichter Schleimüberzug das Eindringen von Salzen zu hindern im Stande ist.

Von einigen gauz jungen Blättern von Brasenia peltata, deren Schleimüberzug noch ganz intakt war, wurden die Blattflächen in eine 10-procentige Kalisalpeterlösung getaucht, wobei die angeschnittenen Blattstiele ausserhalb der Lösung verblieben, um das Eindringen der Letzteren durch die Schnittflächen zu verhindern. Nach 16 Minuten erschienen die Blattspreiten vollkommen schlaff in Folge der entstandenen Plasmolyse, sodass also dieser kurze Zeitraum genügt hatte, um das Hindurchtreten des Kalisalpeters durch die umhüllende Schleimschicht hindurch in das Blattinnere zu ermöglichen. Darauf 
in reines Wasser gebracht, zeigten die Blätter schon nach 23 Minuten wieder ihre frühere Straffheit.

Dies eine Beispiel dürfte wohl genügen, um die Unhaltbarkeit der Schilurng'schen Behauptung darzuthun, die von ihrem Autor ganz allgemein auf Wurzeln, Algen, Hepaticae und Farne, soweit sie mit Wasser in Kontakt treten, ausgedehnt wird.

Nunmehr komme ich zur Besprechung des dritten Punktes, nämlich der „untergeordneten Bedeutung“, die, nach Schiluing, der Schleim „als Schutzmittel vor Tierfrass und Algenbesiedelung“ nur haben kann.

Es ist mir unverständlich, wie Schilling, der auf Seite 352 seiner Arbeit Schenck vorwirft, dass er ohne nähere Begründung sich für die Stahl'sche Ansicht entscheidet, inconsequenter Weise ganz ebenso verfährt; denn wenn er, im Anschluss an Goebel, sich gegen die STAhl'sche Ansicht ausspricht, so thut er dies gleichfalls, ohne auch nur den geringsten Versuch einer experimentellen Beweisführung zu unternehmen. Und die Bedeutung der Schleimbildung in dieser Richtung lässt sich so leicht beweisen!

Als Versuchsobjekt benutyte ich Brasenia peltata. Einige junge Blätter, bei denen der Schleim an der Unterseite noch einen dichten Ueberzug bildete, 
wurden auf einer Hälfte vorsichtig ihres Schleimes beraubt.

Diese halb schleimfreien, halb schleimführenden Blätter wurden in ein Gefäss mit den Versuchstieren, in diesem Fall der gewöhnlichen grossen Wasserschnecke, Limnaea stagnalis, gelegt.

Schon am anderen Tage waren an einem Blatte, und eben an jener Stelle, wo der Schleim entfernt worden war, die Spuren von Tierfrass zu konstatieren; der nächste Tag brachte dann die Entscheidung: an mehreren Blättern nämlich, und zwar nur an den schleimfreien Hälften, waren die unteren Blattflächen angefressen, während die schleimführenden Hälften in keinem einzigen Fall die geringsten Spuren von Tierfrass zeigten.

Ich glaube, dass man danach kaum noch bezweifeln kann, dass dem Schleim hier die Bedeutung eines Schutzmittels gegen die Angriffe von Wasserschnecken oder gegen Tierfrass im Allgemeinen zukommt. Die Schleimtrichome, welche hier, wenn das Blatt ausgebildet ist, verschwinden, haben also bei ihrer Schleimbildung die Aufgabe, das junge Blatt gegen die Angriffe von Tieren zu schützen. Der Schutz ist nur so lange nötig, wie das Blatt nicht im Stande ist, sich mechanisch, und höchst 
wahrscheinlich auch wohl chemisch, gegen diese Angriffe zu wehren.

Zur Bestätigung der letzterwähnten Ansicht diene der folgende Versuch, bei welchem wiederum Brasenia peltata benutzt wurde.

Ich nahm ein ganz junges, noch eingerolltes Blatt mit mächtigem Schleimüberzug und ein ganz ausgebildetes Blatt, bei dem die Schleimtrichome an der Unterseite schon verschwunden waren und das also ganz kahl und schleimfrei war. Beide Blätter schnitt ich mit einem Rasiermesser der Länge nach in gleiche Hälften und brachte dann die vier Objekte wiederum in ein Gefäss mit Limnaea.

Nach zwei Tagen konnte ich feststellen, dass die beiden Hälften des ganz jungen, schleimführenden Blattes von den Schnittflächen aus angefressen waren; dagegen war bei den Hälften des ausgebildeten, schleimfreien Blattes weder an der Unterseite selbst, noch an den Schnittflächen die geringste Spur von Frass zu finden. Hieraus ergiebt sich also, dass bei den ausgebildeten Blättern irgend welche Schutzmittel vorhanden sind, die im jugendlichen Zustande noch fehlen.

So viel zur Kritisierung der älteren Ansichten, denen ich jetzt meine eigene Auffassung über die 
Bedeutung, welche ich dem Schleim bei den Wasserpflanzen neben der Funktion als Schutz gegen Tierfrass zuschreibe, gegenüberstellen werde.

Eine allgemeine und in ihrer Wichtigkeit sehr hervorragende Bedeutung des Schleimes wird, wie dies schon StaнL hervorgehoben hat, in seiner schützenden Wirksamkeit gegen das Eindringen kleiner Tiere und vielleicht auch anderer Feinde $\mathrm{zu}$ suchen sein.

Ausserdem aber dürfte der Schleim ganz allgemein dem Zwecke dienen, die mechanisch noch nicht in genügender Weise geschützten Gewebe, sowohl die Knospen, als auch die jüngsten Blätter, vor mechanischer Verletzung jeder Art zu bewahren.

Die noch zarten, nicht angebildeten Organe besitzen in ihrem schleimigen Ueberzug eine Einrichtung, die es ihnen ermöglicht, von den an sie anstossenden Gegenständen, ohne Nachteil zu erleiden, ab7ugleiten.

Betrachten wir nun auch hier die verschiedenen aufeinanderfolgenden Entwickelungsphasen, so finden wir, dass auch bei den Wasserpflanzen Einrichtungen vorhanden sind, welche in der Phase der Entfaltung dazu dienen, diese zu erleichtern.

Der Schleim, welcher die Knospe gegen mecha- 
nische Verletzungen und Tierfrass schützt, fungiert als Schmiere, welche die Reibungen, die bei der Entfaltung auftreten, zu vermindern im Stande ist.

Beispiele dafür sind in grosser Zahl vorhanden. Die Blätter, welche in der Knospenlage fest eingerollt sind, wie bei Brasenia, Cabomba, Nuphar u. s. w., besitzen in der Schleimbildung, die von Seiten oberflächlicher Trichome ausgeht, ein Gleitmittel, welches die Ausbreitung der Blattspreite erleichtert.

Beteiligen sich an der Knospenlage sogenannte Stipeln, wie bei Nymphaea, Euryale u. s. w., so ist es auffallend, dass die Stipeln immer nur auf derjenigen Seite mit Schleimhaaren besetzt sind, welche dem ihnen zugehörigen Blatte zugekehrt ist.

Die Stipeln aber schliessen die Knospe eng ein, sodass bei der Entfaltung grosse Reibungen zu überwinden sind.

Da diese Letzteren aber bloss an der inneren Seite der Stipeln sich einstellen, so genügt es, wenn nur dort eine Schleimabsonderung vor sich geht.

Ebenso finden wir, ganz in Uebereinstimmung mit den Landpflanzen, auch bei denjenigen Wasserpflanzen, wo eine scheidenförmige Verbreiterung des Blattstielfusses vorliegt, eine ausgeprägte Schleimbildung innerhalb dieser Scheide. 
So z. B. bei Ranunculus fluitans, Caltha palustris, Limnanthemum nymphaeoides, Menyathes trifoliata $\mathrm{u}$. s. w., wo die Scheiden sich rings um die jungen Blätter ziehen, sodass bei dem Herauswachsen solch eines eingeschlossenen Blattes Reibungen entstehen müssen.

Der Schleim tritt hier wiederum auf als Schmiere, und das junge Blatt gleitet aus seiner Scheide hervor, ohne irgendwo Schaden gelitten zu haben. Ja, selbst bei den Monocotylen, bei denen wir, mit Ausnahme von Tradescantia, unter den Landformen eine oberflächliche Schleimbildung niemals nachweisen konnten, ist sie bei den Wasserformen in der Regel zu finden.

Wie Schiling ${ }^{1}$ ) gezeigt hat, sind nämlich die sogenannten "Intravaginalschuppen" von Vallisneria, Hydrocharis, Trianea, Alisma u. s. w. Organe für Schleimabsonderung, womit nicht hehauptet werden soll, dass sie nicht auch noch anderen Verrichtungen dienen mögen.

1) L. c., pag. 333 . 


\section{ABSCHNITT.}

\section{Schlussbemerkungen.}

Eine Arbeit, wie die vorliegende, die für grosse Gruppen des Pflanzenreiches einen einheitlichen biologischen Gesichtspunkt vergleichend durchzuführen sich bestrebte, hat wohl das Recht, zum Schlusse noch einmal über ihr eigentliches Untersuchungsfeld hinauszugreifen und Anknüpfungspunkte in dem anderen grossen Organismenreiche, der Tierwelt, zu suchen.

Haben wir wirklich in der Schleimbildung der Pflanzen einen Gleitmechanismus vor uns, geeignet, die Ortsbewegung, die Bewegungen des Wachstums und der Entfaltung zu unterstützen, so liegt der Gedanke nahe, dass auch bei den Tieren, bei denen die Bewegung zu den wichtigsten Lebensäusserungen gehört, ja eine Existenzbedingung bildet, analoge 
Einrichtungen sich werden auffinden lassen. Wir sahen schon, dass für die freie Lokomotionsfähigkeit im Wasser die oberflächliche Schleimbildung das gleiche, mechanisch unterstützende Moment für Pflanzen und 'Tiere darstellt.

Aber damit sind die Vergleichspunkte nicht erschöpft; denn noch für zwei andere grosse Funktionen des Tierkörpers lässt sich die Rolle des Schleimes als eines bedeutungsvollen Gleitmechanismus in einwandfreier Weise darthun, für die Verdauung nämlich und die Gelenkbewegungen.

Es ist ja bekannt, wie mit dem ersten eingeführten Nahrungsbissen eine starke Speichelsekretion beginnt, deren Schleimmassen die oft trockene Substanz umhüllen und sie unter dem Einfluss der Muskulatur des Mundes zu rundlichen Ballen formen, die durch die gleichfalls durch Schleim schlüpfrig gemachte Speiseröhre zum Magen hinabgleiten können. Im ganzen Verlauf des Darmkanales sind unzählige schleimproducierende Becherzellen angeordnet, die für das abgeführte und verbrauchte Mucin stets neuen Ersatz schaffen und es dem nach abwärts immer konsistenter werdenden Darminhalt ermöglichen, unter der Einwirkung der peristaltischen Bewegungen des Verdauungsrohres vorwärts 
zu gleiten. Wie aber überall, so verbindet sich auch hier mit dem Gleitmechanismus der Schutz gegen mechanische Verletzungen, und jene nicht seltenen Fälle, in denen künstliche Gebisse, Nadeln, Messerklingen, ohne Verwundungen zu erzeugen, das enge, mehr als acht Meter lange Darmrohr passierten, beweisen zur Genüge die hohe Bedeutung, die der Schleim bei dem Transport der Ingesta als Schutzmittel gegen mechanische Verletzungen hat.

Als ein Gleitmechanismus reinster Form tritt uns schliesslich die Schleimbildung in den tierischen Gelenken entgegen; hier kann ihre Bedeutung lediglich und allein in einer Erleichterung der Bewegung gegen einander sich verschiebender Knochenflächen bestehen. Wie die Teile einer Maschine, so gleiten in den Gelenken knöcherne Vorsprünge in knöchernen Gruben; in engem Raurne, bei fest aufeinandergepressten Flächen, müssen forcierte Bewegungen, starke Exkursionen ausgeführt werden, und dass dabei Reibungen denkbar grösster Stärke entstehen müssen, leuchtet ein.

Wird, bei zu langandauernden Bewegungen, der Schleim, diese "Gelenkschmiere", wie man ihn nicht mit Unrecht genannt hat, rascher verbraucht, als er 
sich regeneriert, so ist die Folge eine Erschwerung der Lokomotionsfähigkeit, die schliesslich auch durch den stärksten Willen nicht mehr kompensiert werden kann; versiegt in krankhaften Processen die Schleimproduktion, so fühlt und hört man die Gelenkfächen mit knatterndem Geräusch an einander sich reiben, und Funktionsstörung, ja Funktionsaufhebung ist das Resultat.

Ueberall aber im tierischen Körper, wo auf abnorme Weise Reibungen entstehen, sei es, dass ein aus seiner Pfanne getriebener Gelenkkopf sich an anderer Stelle gegen andere Organe bewegt, sei es durch den Bruch eines Knochens, dessen Fragmente sich nunmehr gegen einander verschieben, sei es schliesslich, dass ein Teil der Haut abnormem äusserem Drucke ausgesetzt ist, sehen wir eine Schleimproduktion auftreten, die, den jeweiligen Verhältnissen genau sich anpassend, nur dem Zwecke dient, die entstehenden Reibungen auf ein Minimum zu reducieren und durch gleichmässige Verteilung äusserer Druck- und Gewalteinwirkungen einen mechanischen Schutz zu gewähren.

Gerade diese, der Tierphysiologie entnommenen Thatsachen scheinen mir für meine Untersuchungen nicht wertlos zu sein, da sie gewisser- 
maassen in grossem Stile und in einer von gleichzeitigen Nebenfunktionen freien Form die biologische Bedeutung der Schleimbildung als eines Gleitmechanismus und eines mechanischen Schutzmittels auf das Deutlichste hervortreten lassen.

Diesem letzteren Umstande ist es aber gerade zuzuschreiben, dass in der Tierphysiologie schon lange die Vorstellung der Gleitfunktion sich eingebürgert hat, während die Pflanzenphysiologie, welche gerade hier mit zeitlich und räumlich in kleinsten Grenzen sich abspielenden Vorgängen zu rechnen hat, bei denen das klare Bild einer funktionellen Bedeutung durch interkurrierende Nebenfunktionen verwischt wird, den Gleiteinrichtungen noch nicht die gebührende Aufmerksamkeit geschenkt hat.

Ich hoffe jedoch, durch die vorliegende Untersuchung den Nachweis erbracht zu haben, dass man auch für die Pflanzen berechtigt ist, in vielen Fällen, wo Bewegungen sich abspielen, seien es aktive Lokomotionen, seien es Bewegungen des Wachstums, beziehungsweise der Entfaltung, Einrichtungen anzunehmen, die diese Bewegungen unterstützen und erleichtern, und dass man, unbeschadet zahlreicher, im Verlaufe dieser Arbeit besprochener Nebenfunk- 
tionen, der oberflächlichen Schleimbildung in grossen Gruppen des Pflanzenreiches die Bedeutung eines Gleitmechanismus und eines mechanischen Schutzmittels zuschreiben muss.

Jena, Botanisches Institut,

2. August 1898. 


\section{LITTERATUR-VERZEICHNISS.}

1. Ambronn, H. und Westermaier, M., Ueber eine biologische Eigenthümlichkeit der Azolla caroliniana Link. Abhandlungen d. Botan. Vereins der Provinz Brandenburg, Berlin, 1880, Jahrg. XXII, pag. 58-61. Taf. III.

2. Ascherson, P., Synopsis der mitteleuropäischen Flora, Bd. I.

3. BARY, A. DE, Vergleichende Anatomie der Vegetationsorgane der Phanerogamen und Farne. Handbuch der physiologischen Botanik, Leipzig, 1877, Bd. III.

4. Bary, A. De, Vergleichende Morphologie und Biologie der Pilze. Handbuch der physiologischen Botanik, Leipzig, 1884, Bd. II.

5. Brefeld, O., Botanische Untersuchungen über Schimmelpilze, Leipzig, 1877, Heft III: Basidiomyceten, I.

6. Douliot, H. Siehe Tieghem.

7. Goeber, K., Grundzüge der Systematik und speciellen Pflanzenmorphologie, Leipzig, 1882.

8. Goeber, K., Pflanzenbiologische Schilderungen.

I. Theil, Marburg, 1889.

II. Theil, Marburg, 1891. 
9. Goebel, K., Archegoniaten-Studien.

I. Die einfachste Form der Moose. Flora, Ergänzungsbd. zum Jahrg. 1892, Bd. 76, pag. 92-104. Taf. VIII-IX.

III. Rudimentäre Lebermoose. Flora, Jahrg. 1893, Bd. 77, pag. 82-104. Taf. II.

IV. Zur Kenntniss der Entwickelung von Riella. Flora, Jahrg. 1893, Bd. 77, pag. 104-108.

V. Die Blattbildung bei den Lebermoosen. Flora, Jahrg. 1893, Bd. 77, pag. 423-459. Taf. VIII-IX.

10. Goeber, K., Morphologische und biologische Studien.

I. Ueber epiphytische Farne und Muscineen. Annales du Jardin botan. de Buitenzorg, 1888, Vol. VII, pag. $1-70$.

IV. Ueber javanische Lebermoose. Annales du Jardin botan. de Buitenzorg, 1891, Bd. IX, pag. 1-40.

11. Goebeler, E., Die Schutzvorrichtungen am Stammscheitel der Farne. (Berliner Inaug.-Dissertation.) Flora, Jahrg. 1886, Bd. 69, n . 29-31. Taf. XI.

12. Guignard, L., Observations sur l'appareil mucifère des Laminariacées. Annales des Sciences naturelles, 1892, Sér. VII, Tom. XV, pag. 1-46.

13. Hanstein, J., Ueber die Organe der Harz- und Schleimabsonderung in den Laubknospen. Botanische Zeitung, 1868, n . 43-46. Taf. XI-XII.

14. Hartig, R., Untersuchungen aus dem forstbotanischen Institut zu München, Berlin, 1883, Bd. III.

15. HaUptrleisch, P., Zellmembran und Hüllgallerte der Desmidiaceae, Greifswald, 1888. (Inaug.-Dissertation.)

16. Hopureister, W., Vergleichende Untersuchungen höherer Kryptogamen, Leipzig, 1851.

17. Hopmeister, W., Beiträge zur Kenntnis der Gefässkryptogamen. Abhandl. d. K. Sächs. Gesellsch. d. Wissensch., Leipzig, 1852.

18. Hooker, Synopsis Filicum, 1863. 
19. Karsten, G., Notizen über einige mexikanische Pflanzen. Berichte d. Deutschen Botan. Gesellsch., Jahrg. 1897, Bd. XV, Heft 1, pag. 10-l6. 'Taf. II.

20. Klebahn, H., Beiträge zur Kenntniss der Auxosporenbildung, I. Rhopalodia gibba (Ehrenb.) O. Müller. Jahrbüch. für wissensch. Botanik, herausgeg. v. Pringsheim, Leipzig, 1896, Bd. 29, pag. 595-652.Taf. X.

21. Klebs, G., Ueber die Organisation einiger Flagellatengruppen und ihre Beziehungen zu Algen und Infusoriengruppen. Untersuchungen aus d. botan. Instit. zu Tübingen, 1883, Bd. I, pag. 233-362.

22. Klebs, G., Ueber Bewegung und Schleimbildung der Desmidiaceae. Biologisch. Centralblatt, Jahrg. 1885, Bd. V, $\mathrm{n}^{\circ} .12$.

23. Klebs, G., Ueber die Organisation der Gallerte bei einigen Algen und Flagellaten. Untersuchungen aus d. botan. Instit. zu Tübingen, 1887, Bd. II, Heft 2, pag. 333-418. Taf. IIl-IV.

24. КӥнN, J., Untersuchungen über die Anatomie der Marattiaceen und anderer Gefässkryptogamen. (Münchener Inaug.-Dissertation.) Flora, Jahrg. 1889, Bd. 72, pag. 457-504. Taf. XVIII-XX.

25. Lagerheim, G., Zur Entwickelungsgeschichte des Hydrurus. Berichte d. Deutschen Botan. Gesellsch., 1888, Bd. VI, pag. 73-85.

26. Leitgeb, H., Untersuchungen über die Lebermoose, 1874-1881.

27. Pringsheim, W., Ueber chemische Niederschläge in Gallerte. Jahrbüch. für wissensch. Botanik, herausgeg. von Pringsheim, Leipzig, 1895, Bd. 28, pag. 1-38. Taf. XXII.

28. Raciborski, M., Die Morphologie der Nymphaeaceen und Cabombeen. Flora, Jahrg. 1894, Bd. 78, pag. 244-279.

29. Raciborski, M., Die Schutzvorrichtungen der Blüthenknospen. Flora, Ergänzungsbd. zum Jahrg. 1895, Bd. 8], pag. 151-194.

30. Raciborskr, M., Die Pteridophyten der Flora von Buitenzorg, Ie Partie de la Flore de Buitenzorg, Leide, 1898. 
31. Regnaud, La vie dans les eaux.

32. Ruge, G., Beiträge zur Kenntniss der Vegetationsorgane der Lebermoose. (Münchener Inaug.-Dissertation.) Flora, Jahrg. 1893, Bd. 77, pag. 279-312. Taf. IV.

33. S SCHS, J., Vorlesungen über Pflanzen-Physiologie, Leipzig, 1882.

34. SADEBECK, R., Die Gefässkryptogamen. Handbuch der Botanik, herausgeg. von Schenk u. A., Breslau, 1881, Bd. I, Abth. 3, pag. $147-326$.

35. Schiffner, V., Hepaticae. Die natürlichen Pflanzenfamilien vion Engler und Prante, Leipzig, 1893-1895, Th. I, Abth. 3, Bogen 1-9, Lief. 91. 92. 112.

36. Schiliıvg, A. J., Anatomisch-biologische Untersuchungen über die Schleimbildung der Wasserpflanzen. Flora, Jahrg. 1894, Bd. 78, pag. $280-360$.

37. Schröter, C., Die Schwebeflora unserer Seen. (Das Phytoplankton.) 99. Neujahrsblatt d. Naturforsch. Gesellschaft in Zürich, 1897.

38. Sснӥтt, F., Bacillariales. Die natürlichen Pflanzenfamilien von ENgler und Prante, Leipzig, 1896, Th. I, A bth. 1, b, Bogen 1-10, Lief. $143-145$.

39. Schwarz, Fr., Die Wurzelhaare der Pflanzen. Ein Beitrag zur Biologie und Physiologie dieser Organe. Untersuchungen aus d. botan. Instit. zu Tübingen, 1883, Bd. I, Heft 2, pag. 135-188. Mit 1 Tafel.

40. Solms-Laubach, Hermann Graf zu, Ueber den Bau und die Entwickelung der Ernährungsorgane parasitischer Phanerogamen. Jahrbüch. für wissensch. Botanik, herausgeg. von Pringsheim, leipzig, 1867-1868, Bd. 6, pag. 509—633. Taf. XXXII-XXXIX.

41. Stahu, E., Pflanzen und Schnecken. Sonder-Abdruck aus der Jenaischen Zeitschrift für Naturwissenschaft und Medizin, Jena, 1888, Bd. XXII, N. F., XV. 
42. Tieghem, Ph. van, Traité de Botanique, 2e édit., Paris, 1891.

43. Tieghem, Ph. van et Douliot, H., Recherches comparatives sur l'origine des membres endogènes dans les plantes vasculaires. Annales des Sciences naturelles - Botanique -, 1888, Sér. VII, Tom. VIII, pag. 1-660. Pl. I-XL.

44. Westermater, M. Siehe Ambronn.

45. Wille, N., Bidrag til Algernes physiologiske anatomi. Svenska Vetenskaps-Akademiens handlingar, Bd. XXI, $\mathrm{n}^{\circ}$. 12. [Siehe Referat im Botan. Centralblatt, 1886, Bd. XXVII, n. 27, pag. 1-6.] 


\section{VITA.}

Am 3. April 1874 wurde ich zu Amsterdam (Holland) als Sohn des Kaufmannes JAN George Hunger geboren und auf die Namen Friegrich Wilhelm Tobias getauft.

Meine beiden Eltern verlor ich, als ich sieben Jahre alt war. Nach ihrem Tode wurde ich in das Haus meines in Leiden wohnenden Vormundes aufgenommen, wo ich auch den ersten Unterricht und meine nachherige höhere Ausbildung erhielt.

Im Studienjahre 1892/93 wurde ich bei der Leidener Universität als Student der Naturwissenschaften eingeschrieben, hörte während der nächstfolgenden $31 / 2$ Jahre naturwissenschaftliche Vorlesungen bei den Herren Professoren van Bemmelen, Franchimont, Hoffmann, Lorentz, Martin und Suringar und arbeitete in deren Laboratorien.

Im Sommer-Semester 1896 siedelte ich nach Jena über, wo ich bei der philosophischen Fakultät der dortigen Universität inskribiert wurde. Ich hörte Vorlesungen bei den Herren Professoren Biedermann, Detmer, Enciken, Haeckel, KüKenthal, Liebmann, Linck, Stahl und WALTHER und arbeitete im zoologischen und botanischen Laboratorium. Auch bekleidete ich im Winter-Semester 1896/97 und im Sommer-Semester 1897 die Stelle eines Assistenten am dortigen botanischen Institut. 


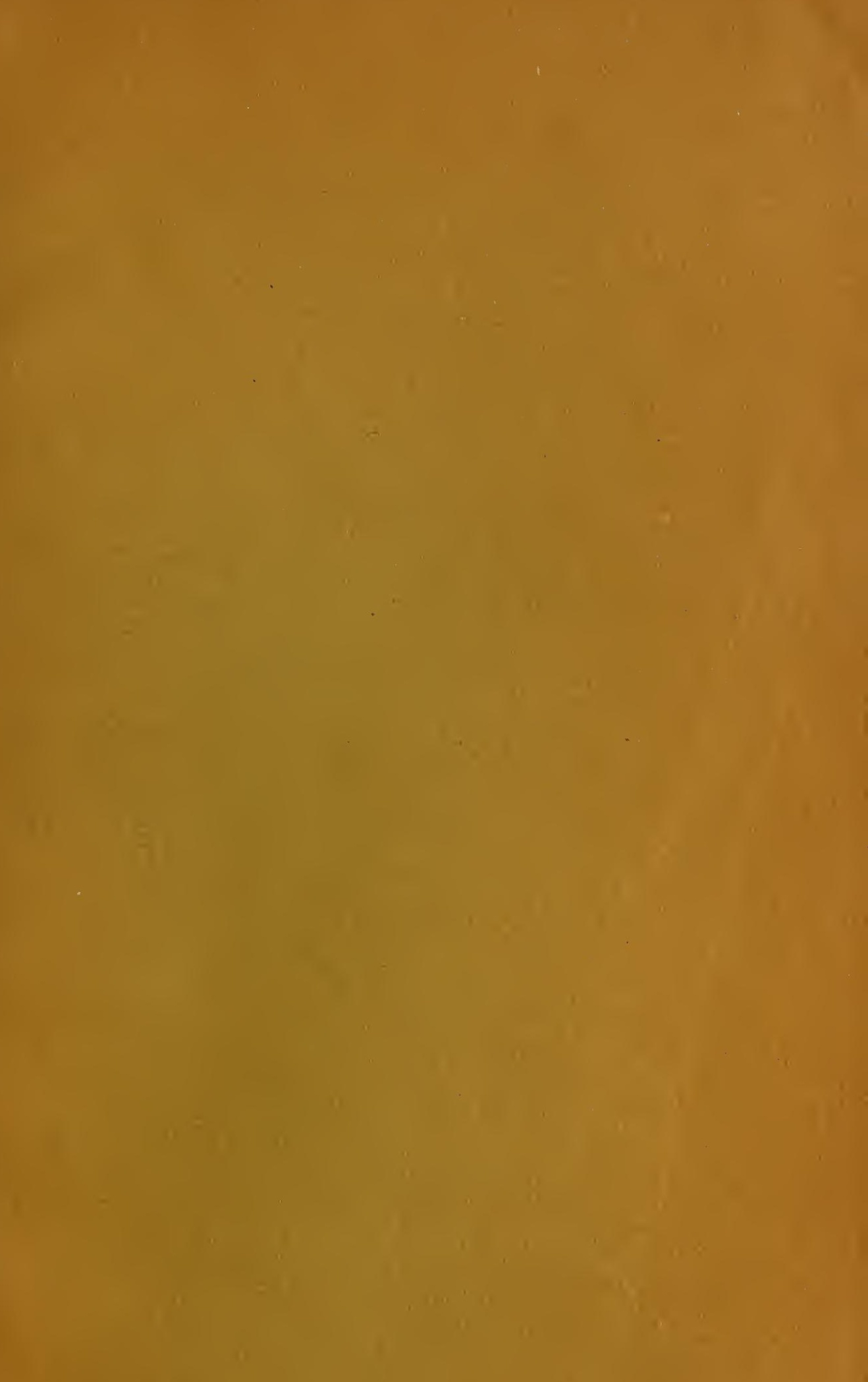


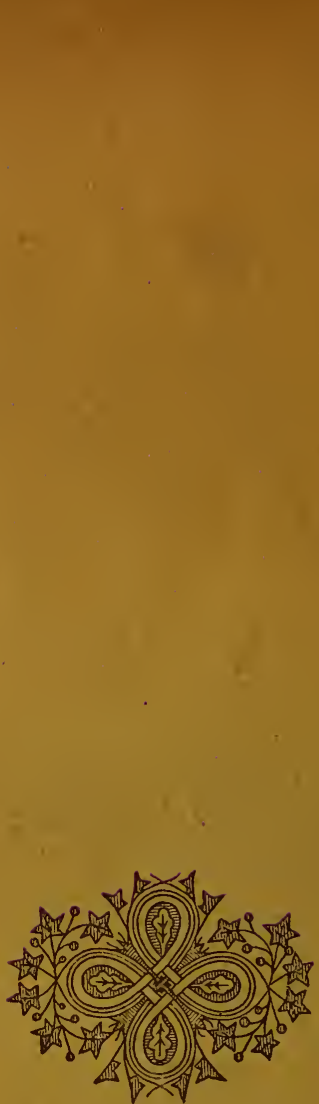



SMITHSONIAN INSTITUTION LIBRARIES

|| ||| ||||||||||||||||||||| |||||||||||||||||||||||| |||||||||||||

|||||||||||||||||||||||||||||||||||||||||||||||||||||||||

3 9088006132021 